\title{
PETROLOGICAL RELATIONS IN SOME ICELANDIC BASALTIC HYALOCLASTITES
}

\author{
HEIKKI MÄKIPÄ
}

\begin{abstract}
MAKIPÄÄ, HEIKKI 1978: Petrological relations in some Icelandic basaltic hyaloclastites. Bull. Geol. Soc. Finland 50: 81-112.

Analyses of a number of subglacially formed basaltic hyaloclastites are used to evaluate various properties of the magmas on eruption. Glass inclusions in olivine and plagioclase crystals have similar composition as the surrounding glass and crystals are therefore true phenocrysts. These data combined with simplified experimental systems can be used to show that crystallization of the lavas studied took place under varying water pressure conditions. The maximum total pressure did not exceed $2 \mathrm{~kb}$. Mineral liquid calculations show that variation between samples from any single area can be explained by fractionation less than $20 \%$ olivine and plagioclase.
\end{abstract}

Heikki Mäkipää, Nordic Volcanological Institute, University of Iceland, Geoscience building, 101 Reykjavik, Iceland

\section{Introduction}

This paper describes the petrography and chemistry of some Pleistocene hyaloclastites in Iceland. These rocks are basaltic magmas quenched in water (mainly glacial melt water) upon eruption. Phenocrysts are therefore unaffected by reactions occuring upon cooling and crystallization.

Iceland provides an unique opportunity to study naturally quenched and completely fresh basaltic rocks. This offers the possibility to study the composition and homogenity of crystals and liquids and conclusions can be drawn about the state of equilibrium.

Analytical data on 22 samples are presented. They are quartz- and olivine-normative tholeiites not far from the normative quartz saturation plane. All samples studied have phenocrysts of olivine, plagioclase and most often clinopyroxene, which in general appear to be in equilibrium with the coexisting liquids. Xenocrysts, clearly out of equilibrium under any conditions, are found in basalts from near the central volcanoes and can be explained as contamination from acid rocks.

The chemical variation in the basalts is mainly discussed on the basis of Ab-An-DiFo- and CMAS-systems. The main result is that the total pressure of the main crystallization does not exceed $2 \mathrm{~kb}(\sim 6-7 \mathrm{~km})$. The derived water pressures vary regionally. Estimates of original water contents, using glass inclusion chemistry in crystals, indicate that those basalts which equilibrated at the 


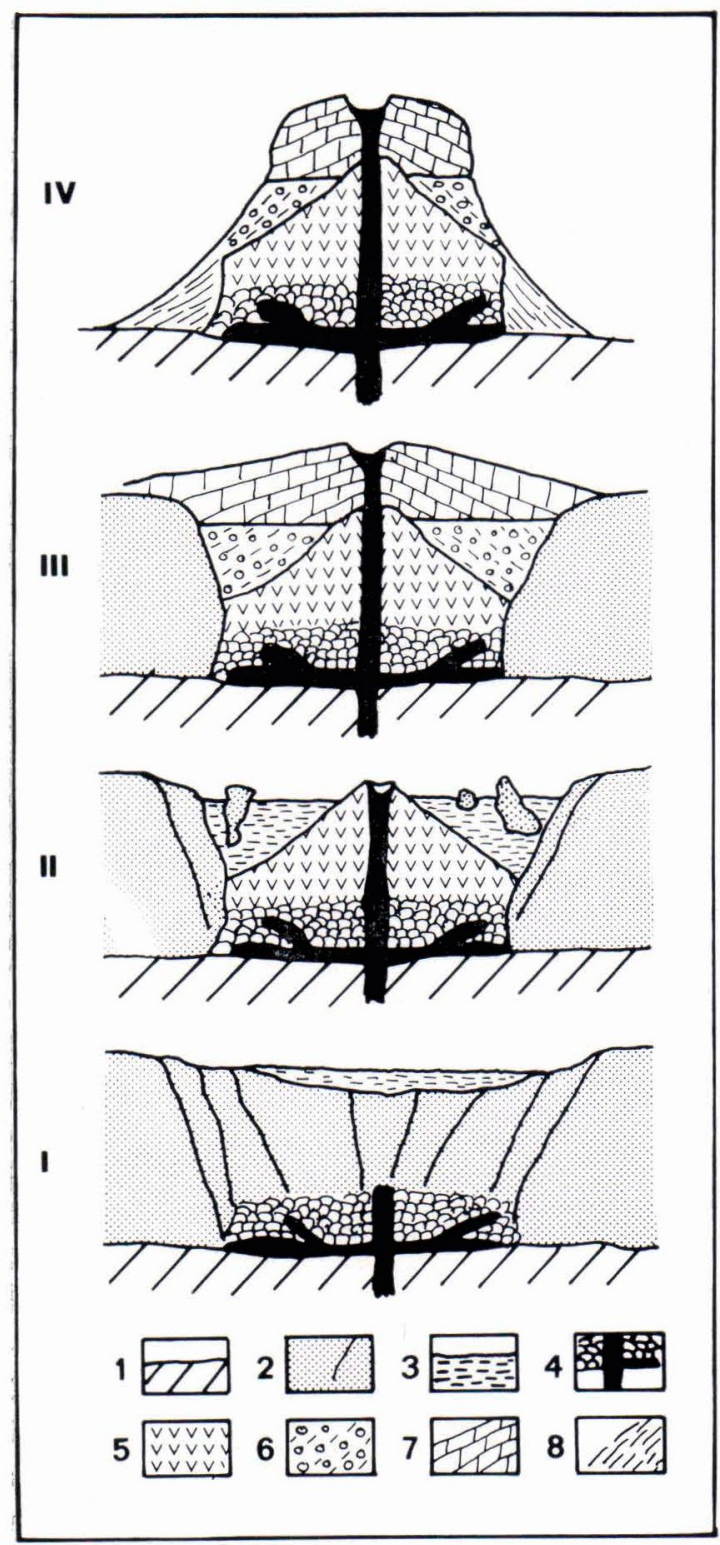

Fig. 1. The building up of hyaloclastite ridge or a table mountain (Einarsson 1968). 1. Bed rock, 2. Glacier, 3. Melt water, 4. Pillow lava and dikes, 5. Tuff and breccia, 6. Foreset bedded pillowbreccia, 7. Lava, 8. Scree.

highest assumed water pressure also have the highest water content.

The model calculations demonstrate that the basalts from the same area can be interrelated by a process of fractional crystalli- zation. However, due to limited data no further attempt has been made to evaluate the origin and evolution of these basalts.

\section{Petrology of hyaloclastites}

\section{General}

The origin of the hyaloclastites has been discussed extensively in the literature. At an early state (e.g. Pjetruss 1900; Thoroddsen 1906) these formations were believed to be remnants of larger volcanoes affected by erosion and faulting.

However, after 1920 (Peacock 1926a, 1926b, Noe-Nygaard 1940, Kjartansson 1943) it was recognized that the hyaloclastite tuffs (palagonite formation or moberg formation) are of subglacial or subaquatic origin and formed by rapid chilling of the magma in contact with water.

In the previous mentioned works (e.g. Peacock 1926a, Noe-Nygaard 1940) the terms palagonite or moberg formations were used. Rittman (1961) proposed a new term 'hyaloclastite' for primary palagonites. The term palagonite in Iceland has been extended to all kinds of deposits which include fragmented subglacial basaltic lavas, glacial moraines, tuffs and alluvial deposits (Nayudu 1964).

The hyaloclastite term in the present study is used genetically and includes the products of subglacial or subaquatic eruptions. The present author does not treat the structures of subglacial formations (but rather the mineralogical problems) but a short description is given for some of them.

The subaquatic volcanic piles have a regular structural sequence (Fig. 1) consisting of pillow lavas at the base, covered by pillow breccias and finally glassy tuffs (e.g. Noe-Nygaard 1940, Bemmelen and Rutten 1955, Sigvaldason 1968, Jones 1970).

The following short definition is cited from Grönvold (1972). 


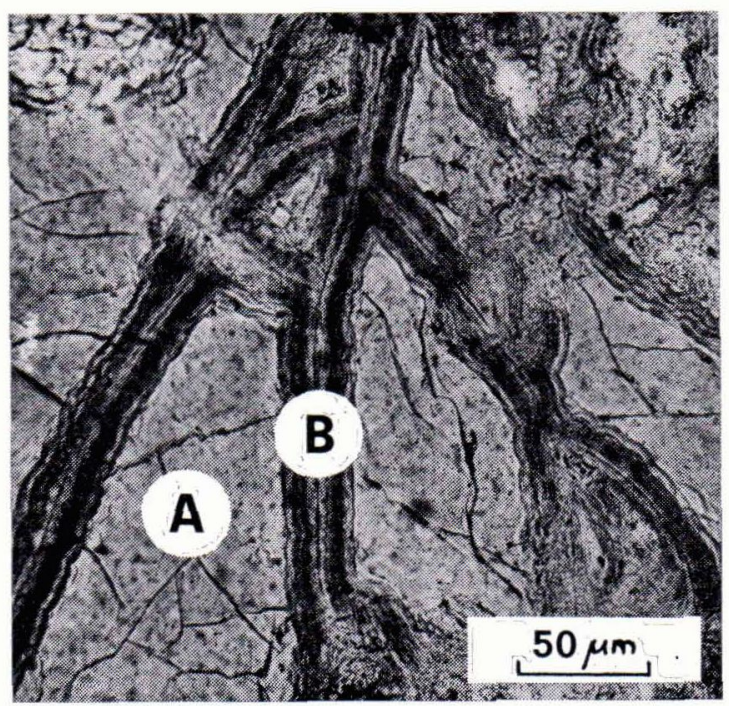

Fig. 2. Palagonitization of glass, Sveifluháls. $A=$ sideromelane glass, $\mathrm{B}=0.01-0.02 \mathrm{~mm}$ wide palagonitization rim.

Pillow lava: Used in the conventional sense for basaltic and intermediate rocks. There is a considerable variation in the size of the pillows, their regularity and the relative amount of the interpillow matrix.

Pillow-breccia: In some cases breccias are so obviously formed from fragments of broken pillows that they are given this special name. The clasts in pillow breccias are characteristically elongated and angular and frequently one side has a glassy skin. Hyaloclastite tuff or glassy tuff: Fine grained basaltic or andesitic rock. Made dominantly of small very vesicular sideromelane (brown glass) fragments, sometimes altered to palagonite.

The subaquatic basalts studied here show many common textural and petrographical features. Peacock (1926a) and Tyrrel and Peacock (1926) made detailed microscopic studies of the tuffs. They subdivided the glasses into two types; sideromelane which is clear, colourless to yellowish brown, and tachylite which is opaque and clouded with minute ore grains. The glass may then alter

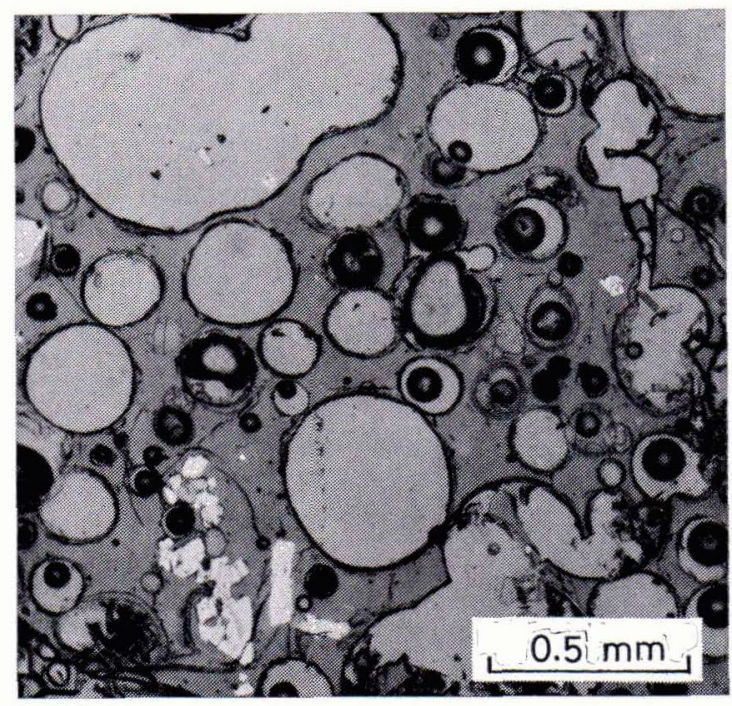

Fig. 3. Vesicular single glass shard (containing scattered euhedral phenocrysts) from a relatively coarse grained hyaloclastite tuff of olivine normative tholeiite (RE-19).

after deposition. The initial alteration product is palagonite. Palagonitization is mainly a hydration process at a relatively low temperature (Peacock 1926a, Hoppe 1941, Furnes 1974, 1975).

Jakobsson (1971) studied the hyaloclastites of Surtsey and showed that considerable amount of $\mathrm{SiO}_{2}, \mathrm{Al}_{2} \mathrm{O}_{3}, \mathrm{CaO}, \mathrm{Na}_{2} \mathrm{O}$ and $\mathrm{K}_{2} \mathrm{O}$ are leached from the glass during palagonitization, $\mathrm{H}_{2} \mathrm{O}$ is absorbed and $\mathrm{Fe}$ is oxidized to give the glass a rust brown colour (Fig. 2).

Furnes $(1974,1975)$ studied experimentally the palagonitization process and demonstrated the same effect as described by Jakobsson (1971).

The rocks studied range from fine grained $(\varnothing \mathrm{mm})$ to coarse grained $(\varnothing \mathrm{cm})$. The quartz normative rocks are usually much more fine grained than the olivine normative ones (Figs. 3 and 4). This probably reflects different water content and viscosity. The rocks studied have also high amount of vesicles up to $60-70 \%$. Several authors (e.g. Jones 1969) have tried to use the vesicularity as a depth indicator. 


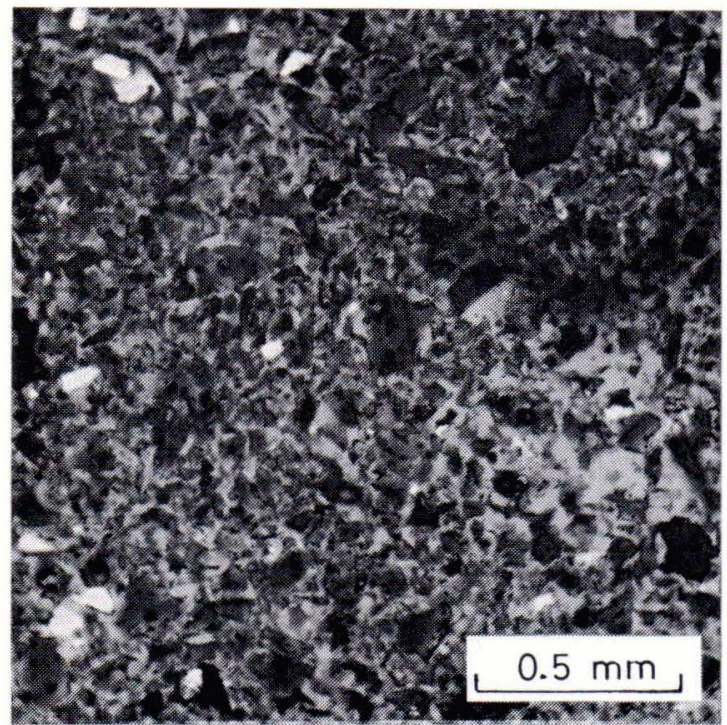

Fig. 4. Fine grained hyaloclastite tuff of quartz normative tholeiite (containing few anhedral xenocrysts) (GR-22).
Mineral phases identified in the glasses are olivine, plagioclase, clinopyroxene, albite, pigeonite, spinel and some iron oxides. Olivine, plagioclase, clinopyroxene and spinel form the phenocryst phases. In all samples olivine and plagioclase occur as phenocrysts, but some rocks contain only a very few crystals. The principal mineralogical difference between individual samples is the variation in the total amount of olivine and plagioclase phenocrysts.

All the phenocrysts are divided into microphenocrysts $(\varnothing<30 \mu \mathrm{m})$ and less abundant macrophenocrysts $(\varnothing>30 \mu \mathrm{m})$.

The sample locations are shown in Fig. 5. The criteria used for the sample selection were 1) to get as representative series of samples from all the tholeitic volcanic zones

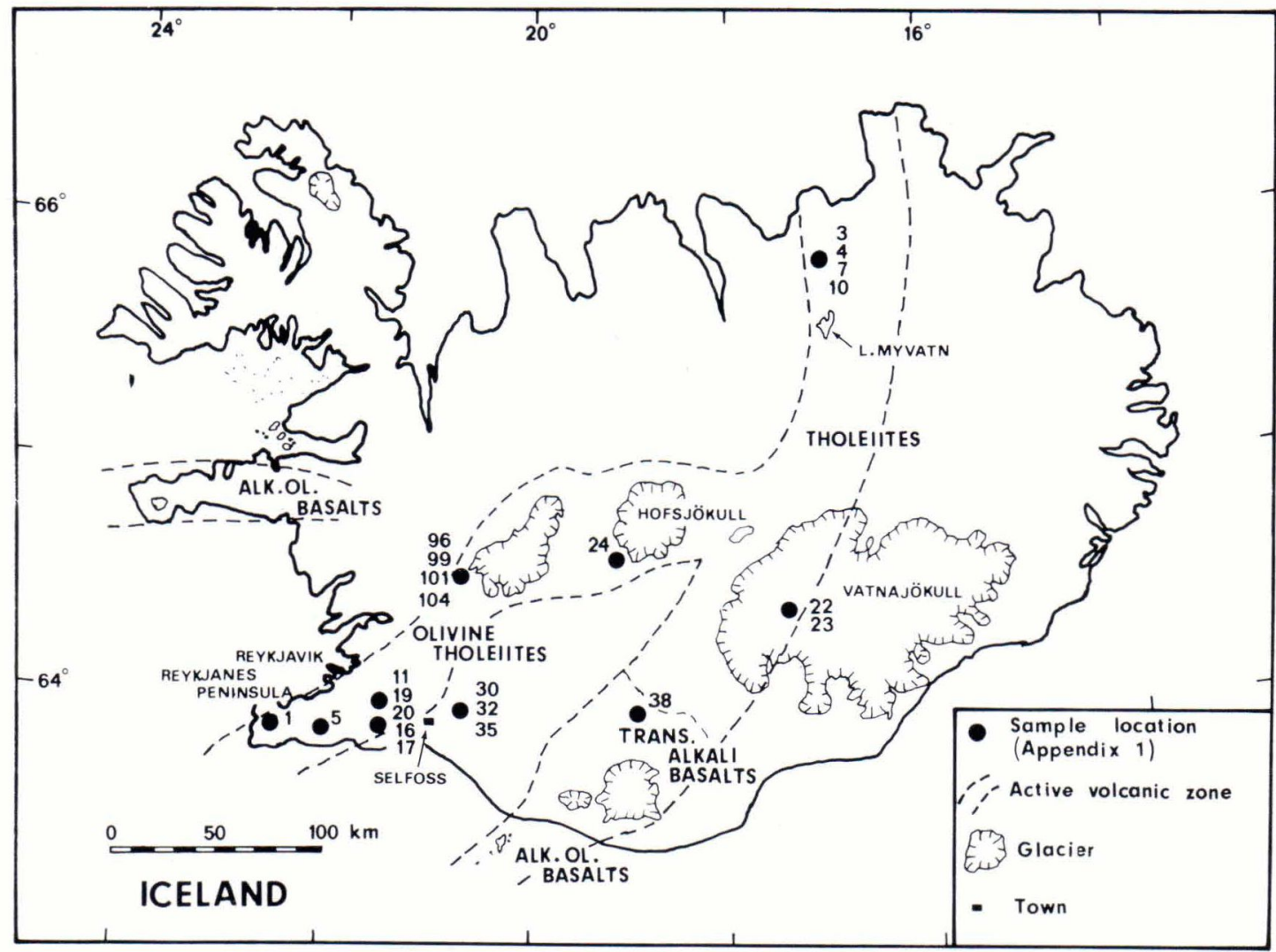

Fig. 5. Sketch map of Iceland showing the location of the active volcanic zones, the location of samples and the petrological basalt types erupted within volcanic zones (after Jakobsson 1972). 
Fig. 6. Plot of the analyses on the AFM diagram. $\bullet=$ whole rock analyses (Table 1). $\mathrm{O}=$ glass analyses (Table 2). $\mathrm{A}=$ $\mathrm{Na}_{2} \mathrm{O}+\mathrm{K}_{2} \mathrm{O}, \quad \mathrm{F}=\mathrm{FeO}+\mathrm{F}_{2} \mathrm{O}_{3}, \quad \mathrm{M}$ $=\mathrm{MgO}$.

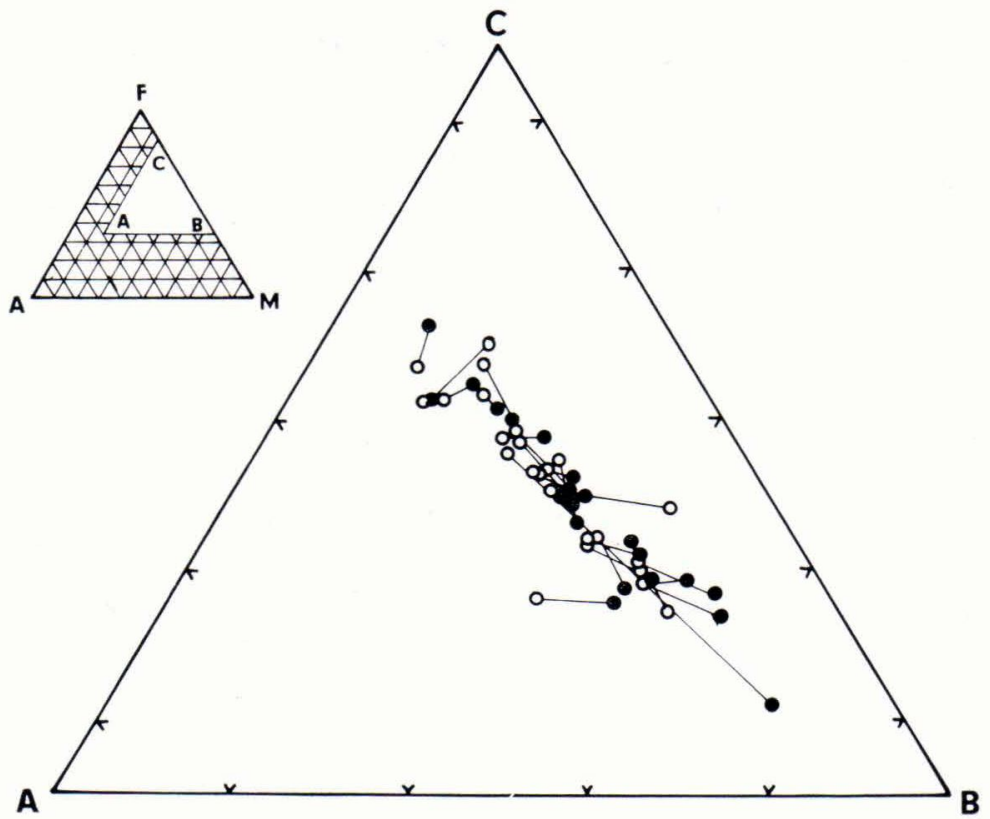

of Iceland as possible and 2) to use only fresh rocks where no palagonitization can be found. Also all breccia types were excluded. All the samples used here are briefly described in Appendix 1.

\section{Analytical methods}

All mineral and glass analyses were carried out on an automatic ARL-SEMQ microprobe.

The hyaloclastites were mounted directly in epoxy for analyses. For whole rock analysis about $100 \mathrm{mg}$ of sample powder were melted in a $\mathrm{Pt}$ loop in a furnace at $1315^{\circ} \mathrm{C}$ for 2 hours with oxygen fugacity close to the QMF-buffer. The sample was quenched in water and the glass was mounted and analyzed in the same way as natural glasses.

Major element analyses were obtained by operating the electron probe at an accelerating potential of $15 \mathrm{kV}$ and $25 \mathrm{nA}$ sample current. Counting time of 20 seconds on peak and 4 seconds on background was used.

The correction procedure of Bence and
Albee (1968) was used and the correction factors of Albee and Ray (1970). Data for all standards are available at the Nordic Volcanological Institute (Imsland 1978).

$\mathrm{FeO}$ in the glass fraction (separated by bromoform) was determined by the modified Wilson method, in the quenched glass the $\mathrm{Fe}_{2} \mathrm{O}_{3} / \mathrm{FeO}$ is assumed to be 0.15 .

Trace element analyses were carried out using $25 \mathrm{kV}$ accelerating potential and 60 $\mathrm{nA}-0.6 \mu \mathrm{A}$ sample current. The detection limit depends on the element and the matrix but 100 ppm detection limit is typical unless special operating conditions are employed. Counting times used were 100 seconds and 10 seconds on peak and background, respectively. The X-ray peak intensities were corrected for background intensity only.

Standards employed for the various trace elements were as follows: augite and hornblende (Mason and Allen 1973) for all transition metals in low concentrations. In high concentrations the standards were: $99.99 \%$ pure metals for $\mathrm{V}, \mathrm{Co}$ and $\mathrm{Ni}$, rhodonite for $\mathrm{Mn}$ and $\mathrm{Zn}$ and synthetic calchopyrite for $\mathrm{Cu}$. 


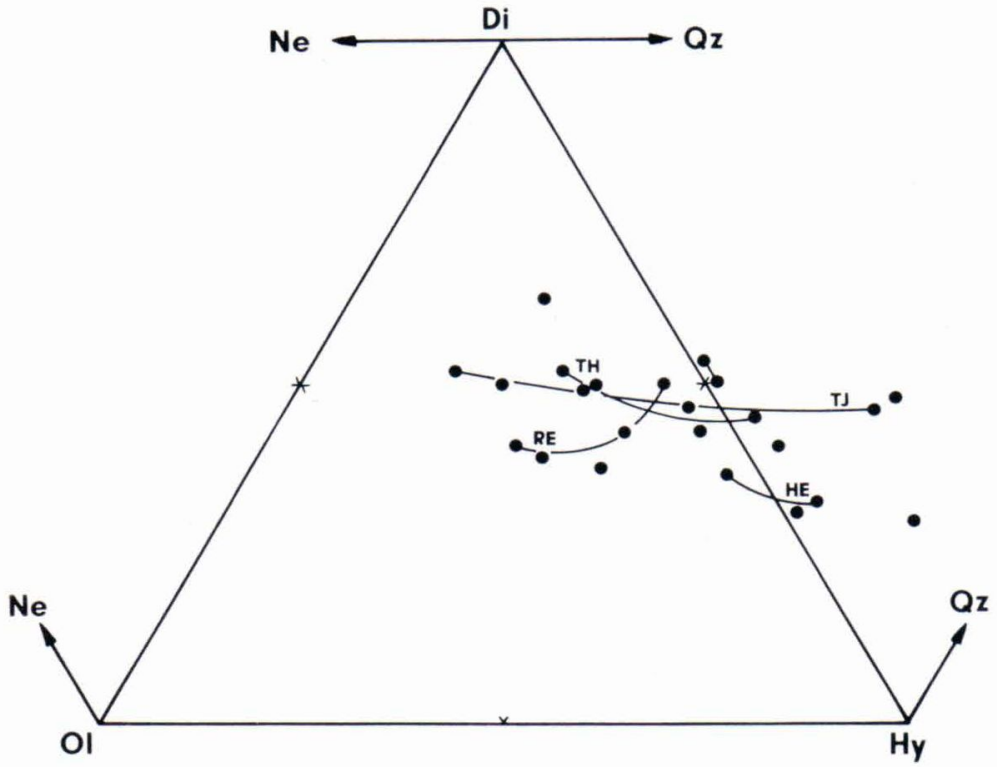

Fig. 7. Plot of the whole rock analyses (Table 1) on the normative Qz-Di-Hy-Ol-Ne diagram. TH - Theistareykir area (No. 3 in Fig. 5). TJ - Thorisjökull area (No. 96 in Fig. 5). Re - Reykjanes peninsula (No. 5 in Fig. 5). HE - Hestfjall (No. 30 in Fig. 5). The curves indicate the end members of separated spatial groups.
Whole rock analysis

The samples were collected from all the tholeiitic volcanic zones of Iceland (Fig. 5). The major element chemistry of the rocks studied follows remarkably well the geographical pattern of the rock type distribution described by Jakobsson (1972).

The whole rock analyses are listed in Table 1 and plotted on the AFM diagram (Fig. 6). Each reported value is the average of many (up to 20) spot analyses which all vary within the range of the analytical standard deviations.

In the normative Qz-Di-Hy-Ol-Ne diagram (Fig. 7) all the samples are very near the silica saturation plane, which divides the quartz and olivine normative tholeiites.

The trace element data including all transition metals (except $\mathrm{Sc}$ and $\mathrm{Fe}$ ) is given in Table 1. Because some elements are strongly affected by fractionation of a specific phase (e.g. $\mathrm{Ni}$ and $\mathrm{Co}$ in olivine and $\mathrm{V}$ in clinopyroxene), they provide a sensitive test of fractional crystallization models.
Glass analysis

The natural glass analyses are listed in Table 2 and plotted on the same AFM diagram (Fig. 6) with the whole rock compositions to show the effect of crystallization.

Mineral analysis

Olivine. Olivine is present in all samples. Commonly the olivine crystals are very small (approximately $5-30 \mu \mathrm{m}$ in diameter) and only few samples contain larger crystals. The olivine phenocrysts show a variety of crystal forms (Fig. 8). Usually microphenocrysts are euhedral but a subhedral shape is common. As seen in many Mid-Atlantic ridge basalts the olivine phenocrysts show varying degree of irregular and lattice-like growth (Bryan 1972). Every sample of this study shows evidence of similar growth.

Analyses of olivines are listed in Table 3. The Fo-contents vary between 65.0 and 89.0 mole- $\%$. 

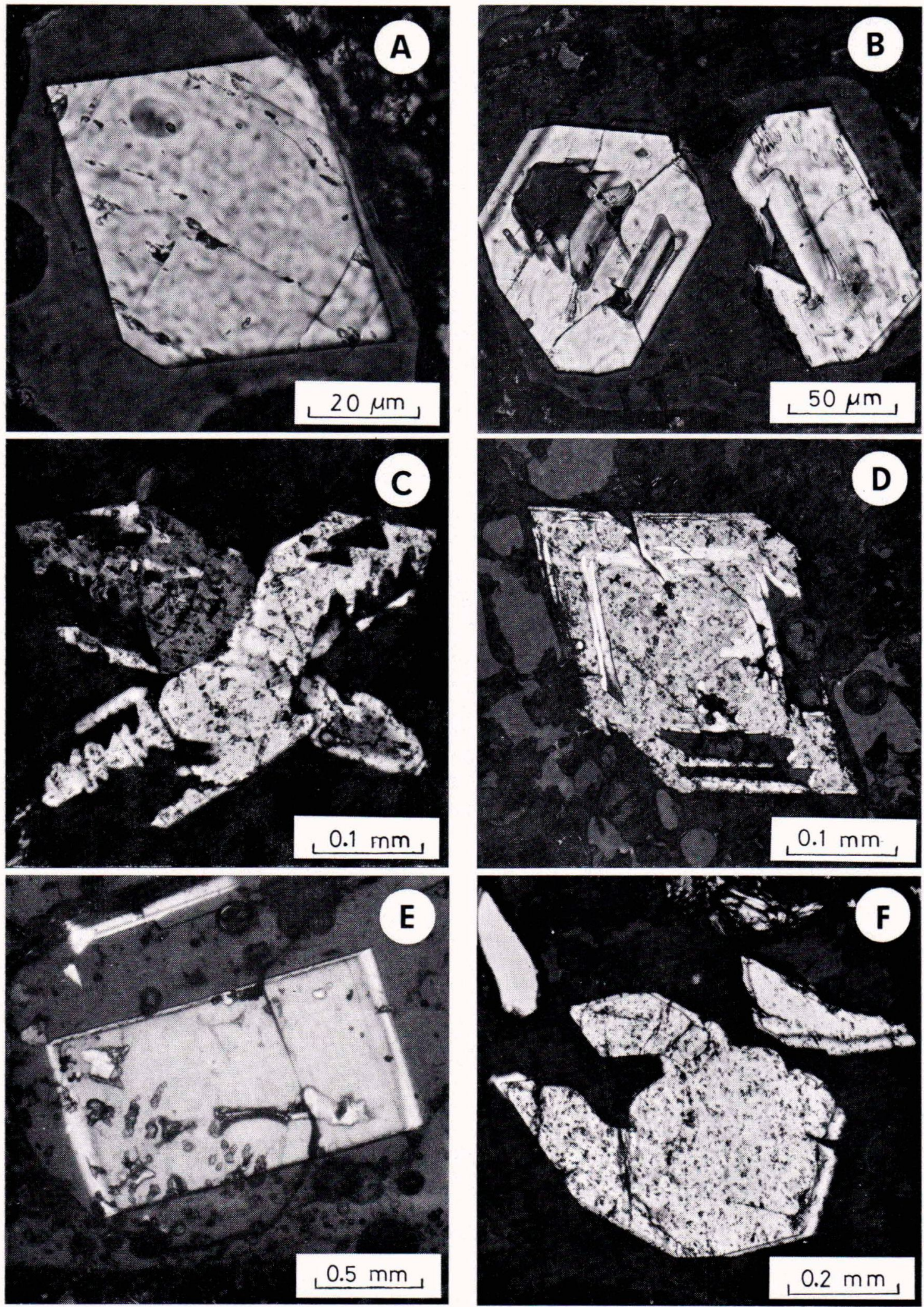

Fig. 8. Olivine morphologies. A euhedral olivine microphenocryst (RE-17), B subheral hollow olivine (HE-30), C subhedral lantern-shaped olivines (HE-35), D subhedral lantern-shaped olivine (HE-32), E euhedral olivine macrophenocryst (RE-17), F skeletal olivine (tabular shaped) (RE-17) 
Table 1. Microproble analyses and CIPW norms of hyaloclastites. Whole rock analyses. Sample location is shown on the map (Fig. 5) and given in Appendix 1.

\begin{tabular}{|c|c|c|c|c|c|c|c|c|c|c|c|c|}
\hline \multirow{2}{*}{\multicolumn{3}{|c|}{$\begin{array}{l}\text { Sample No. } \\
\text { No- of anal. } \\
\text { No }\end{array}$}} & TH-3 & $\mathrm{TH}-4$ & $\mathrm{RE}-5$ & TH-7 & TH-10 & $\mathrm{RE}-11$ & $\mathrm{RE}-16$ & RE-17 & $\mathrm{RE}-19$ & RE-20 \\
\hline No. of anal. & & & 15 & 15 & 18 & 15 & 15 & 18 & 15 & 18 & 15 & 15 \\
\hline \multicolumn{2}{|c|}{$\mathrm{SiO}_{2}$} & 49.23 & 49.64 & 49.05 & 48.98 & 49.01 & 49.26 & 48.26 & 47.75 & 48.44 & 46.89 & 47.50 \\
\hline \multicolumn{2}{|c|}{$\mathrm{TiO}_{2}$} & 1.62 & 1.91 & 1.35 & 1.63 & 1.96 & 1.34 & 1.07 & 1.40 & 1.51 & 1.60 & 1.54 \\
\hline \multirow{2}{*}{\multicolumn{2}{|c|}{$\mathrm{Al}_{2} \mathrm{O}_{3}$}} & 14.57 & 14.12 & 15.48 & 14.93 & 14.29 & 14.24 & 14.95 & 17.61 & 15.89 & 14.69 & 14.94 \\
\hline & & 12.32 & 13.98 & 10.31 & 12.14 & 13.80 & 11.85 & 9.36 & 10.68 & 10.91 & 12.06 & 11.59 \\
\hline \multicolumn{2}{|c|}{$\mathrm{MnO}$} & 0.19 & 0.21 & 0.17 & 0.20 & 0.21 & 0.19 & 0.15 & 0.16 & 0.18 & 0.20 & 0.21 \\
\hline \multicolumn{2}{|c|}{$\mathrm{MgO}$} & 7.89 & 6.59 & 8.50 & 7.39 & 6.33 & 7.09 & 11.93 & 6.63 & 6.87 & 9.47 & 9.76 \\
\hline \multicolumn{2}{|c|}{$\mathrm{CaO}$} & 11.28 & 10.66 & 12.22 & 11.38 & 10.14 & 10.66 & 11.56 & 11.84 & 11.95 & 10.79 & 10.80 \\
\hline \multicolumn{2}{|c|}{$\mathrm{Na}_{2} \mathrm{O}$} & 2.41 & 2.51 & 2.15 & 2.39 & 2.55 & 1.99 & 1.44 & 2.05 & 2.08 & 2.02 & 2.03 \\
\hline \multicolumn{2}{|c|}{$\mathrm{K}_{2} \mathrm{O}$} & 0.20 & 0.23 & 0.18 & 0.20 & 0.22 & 0.18 & 0.10 & 0.16 & 0.17 & 0.16 & 0.15 \\
\hline \multicolumn{2}{|c|}{$\mathrm{P}_{2} \mathrm{O}_{5}$} & 0.20 & 0.25 & 0.14 & 0.19 & 0.22 & 0.19 & 0.12 & 0.16 & 0.15 & 0.17 & 0.19 \\
\hline \multicolumn{13}{|c|}{ CIPW norms (with fixed $\mathrm{Fe}_{2} \mathrm{O}_{3} / \mathrm{FeO}=0.15$ and recalculated to $100 \%$ ) } \\
\hline \multicolumn{2}{|l|}{ Qz } & - & - & - & - & - & 1.80 & - & - & - & - & - \\
\hline \multicolumn{2}{|l|}{ Or } & 1.18 & 1.35 & 1.07 & 1.19 & 1.31 & 1.09 & 0.60 & 0.96 & 1.02 & 0.96 & 0.89 \\
\hline \multicolumn{2}{|l|}{$\mathrm{Ab}$} & 20.38 & 21.15 & 18.24 & 20.28 & 21.81 & 17.32 & 12.29 & 17.59 & 17.90 & 17.37 & 17.34 \\
\hline \multicolumn{2}{|l|}{ An } & 28.33 & 26.47 & 32.14 & 29.51 & 27.19 & 30.24 & 34.33 & 38.92 & 34.08 & 31.05 & 31.51 \\
\hline \multirow{3}{*}{$\mathrm{Di}$} & Wo & 10.98 & 10.26 & 11.58 & 10.80 & 9.27 & 9.56 & 9.49 & 8.18 & 10.52 & 9.28 & 8.91 \\
\hline & En & 5.85 & 4.85 & 6.76 & 5.67 & 4.30 & 4.92 & 6.21 & 4.29 & 5.60 & 5.36 & 5.26 \\
\hline & Fs & 4.78 & 5.28 & 4.27 & 4.82 & 4.88 & 4.39 & 2.61 & 3.65 & 4.59 & 3.50 & 3.20 \\
\hline \multirow{2}{*}{ Hy } & En & 8.52 & 10.40 & 8.31 & 8.48 & 10.54 & 13.25 & 14.72 & 8.76 & 10.20 & 8.36 & 9.57 \\
\hline & Fs & 6.97 & 11.31 & 5.25 & 7.23 & 11.95 & 11.82 & 6.19 & 7.44 & 8.35 & 5.46 & 5.82 \\
\hline \multirow{2}{*}{ Ol } & Fo & 3.69 & 0.77 & 4.31 & 3.02 & 0.76 & - & 6.34 & 2.57 & 1.12 & 7.18 & 6.80 \\
\hline & $\mathrm{Fa}$ & 3.33 & 0.92 & 2.10 & 2.83 & 0.95 & - & 2.94 & 2.41 & 1.01 & 5.17 & 4.56 \\
\hline \multicolumn{2}{|l|}{ Il } & 3.17 & 3.99 & 2.76 & 3.39 & 2.87 & 2.81 & 2.18 & 2.77 & 3.13 & 3.55 & 3.43 \\
\hline \multirow{2}{*}{\multicolumn{2}{|c|}{$\mathrm{Ap}$}} & 2.26 & 2.67 & 1.98 & 2.33 & 2.67 & 2.34 & 1.81 & 2.08 & 1.55 & 1.70 & 2.24 \\
\hline & & 0.46 & 0.58 & 0.33 & 0.44 & 0.52 & 0.45 & 0.28 & 0.38 & 0.35 & 0.40 & 0.44 \\
\hline \multicolumn{13}{|c|}{ Transition metals (except $\mathrm{Sc}$ and $\mathrm{Fe}$ ) in ppm } \\
\hline \multicolumn{2}{|l|}{$\mathrm{Ti}$} & 9695 & 11470 & 8075 & 9765 & 11750 & 8030 & 6400 & 8420 & 9035 & 9580 & 9255 \\
\hline \multicolumn{2}{|l|}{$\mathrm{V}$} & 255 & 260 & 185 & 270 & 260 & 240 & 145 & 195 & 315 & 310 & 255 \\
\hline $\mathrm{Cr}$ & & 160 & 70 & 275 & 160 & 65 & 75 & 385 & 135 & 125 & 255 & 270 \\
\hline Mn & & 1510 & 1640 & 1300 & 1550 & 1605 & 1450 & 1160 & 1260 & 1410 & 1565 & 1615 \\
\hline Co & & 85 & 85 & 120 & 65 & 35 & 105 & 90 & 60 & 140 & 115 & 100 \\
\hline $\mathrm{Ni}$ & & 145 & 70 & 300 & 200 & 135 & 165 & 320 & 155 & 295 & 320 & 420 \\
\hline $\mathrm{Cu}$ & & 70 & 145 & 65 & 90 & 70 & 145 & 180 & 80 & 110 & 90 & 160 \\
\hline $\mathrm{Zn}$ & & 20 & 50 & 25 & 45 & 80 & 55 & 30 & 25 & 35 & 30 & 35 \\
\hline $\mathrm{FeO}^{+}$ & $\mathrm{MgO}$ & 1.56 & 2.12 & 1.21 & 1.64 & 2.18 & 1.67 & 0.78 & 1.61 & 1.59 & 1.27 & 1.19 \\
\hline $\mathrm{Cr} / \mathrm{N}$ & & 1.10 & 1.00 & 0.92 & 0.80 & 0.48 & 0.45 & 1.20 & 0.87 & 0.42 & 0.70 & 0.64 \\
\hline
\end{tabular}

cont.

All microphenocrysts are homogenous and unzoned. In some macrophenocrysts a slight zoning (less than 2 mole- $\%$ Fo) is found, both normal and occasionally reverse. The liquidcrystal boundary was also studied and a com- positional gradient is no wider than about 15 $\mu \mathrm{m}$ from the olivine-glass interface.

All the analyzed olivine crystals are probably phenocrysts but in two samples (KE-24 and LA-38) anhedral olivines may be xeno- 
(Table 1 continued).

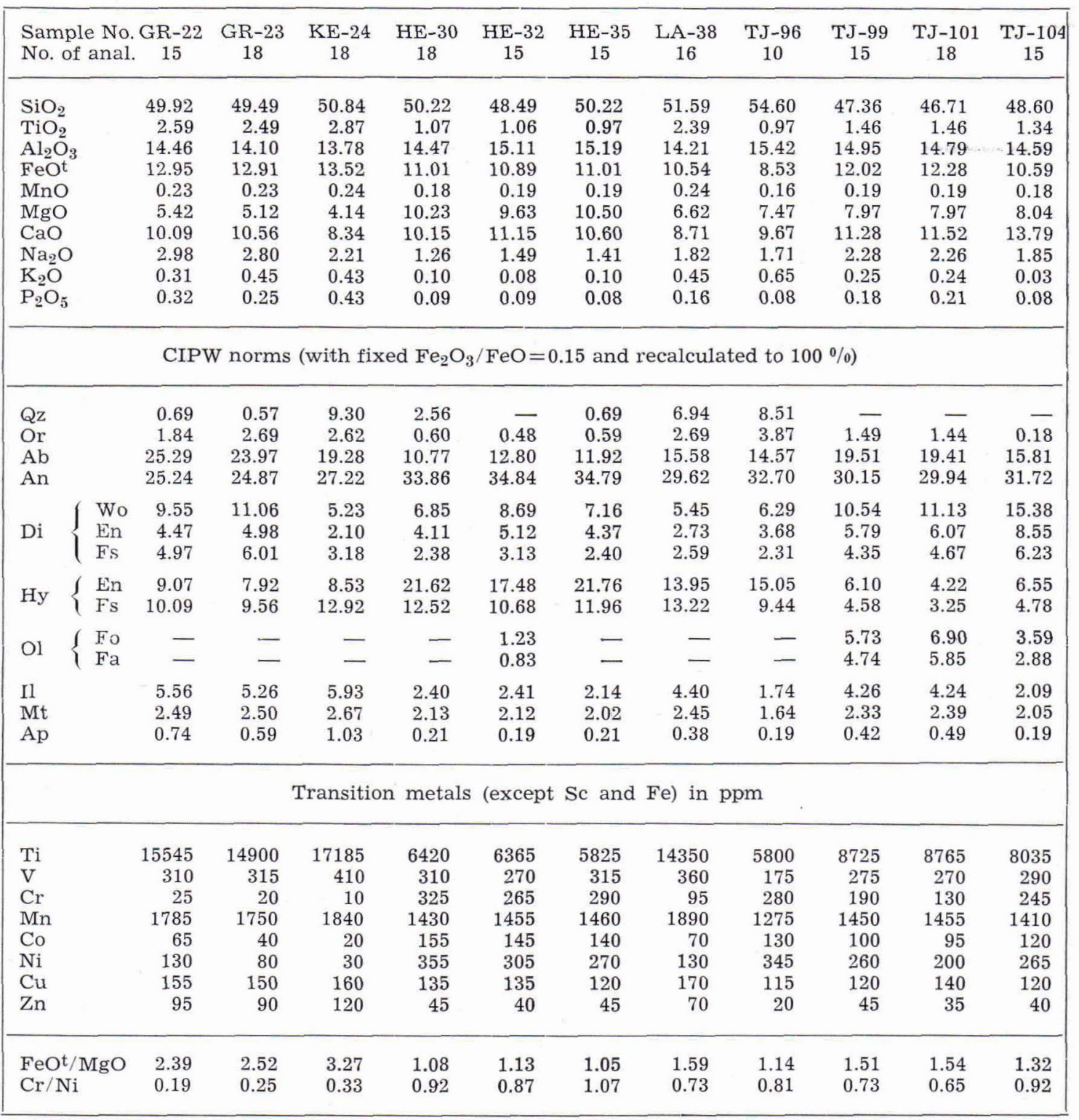

lithic judged by their abnormal calcium content (Fig. 9) (Simkin and Smith 1970).

Plagioclase. Analyses of plagioclase microand macrophenocrysts are listed in Table 4. The anorthite contents vary between 57.9 and 87.0 mole- $\%$.

The small plagioclase laths are homogenous and unzoned. The large phenocrysts are strongly zoned and a variety of zoning types can be found. 
Table 2. Microprobe analyses and CIPW norms of hyaloclastites. Glass phase analyses. Sample location is shown on the map (Fig. 5) and given in Appendix. 1.

\begin{tabular}{|c|c|c|c|c|c|c|c|c|c|c|c|c|c|}
\hline \multicolumn{2}{|c|}{$\begin{array}{l}\text { Sample No. } \\
\text { No. of anal. }\end{array}$} & $\begin{array}{c}\mathrm{RE}-1 \\
20\end{array}$ & $\underset{7}{\text { TH-3 }}$ & $\begin{array}{c}\text { TH-4 } \\
11\end{array}$ & $\begin{array}{c}\text { RE-5 } \\
7\end{array}$ & $\begin{array}{c}\text { TH-7 } \\
12\end{array}$ & $\begin{array}{c}\text { TH-10 } \\
10\end{array}$ & $\begin{array}{c}\text { RE-11 } \\
9\end{array}$ & $\begin{array}{c}\text { RE-16 } \\
16\end{array}$ & $\begin{array}{c}\mathrm{RE}-17 \\
8\end{array}$ & $\begin{array}{c}\text { RE-19 } \\
9\end{array}$ & $\begin{array}{c}\mathrm{RE}-20 \\
9\end{array}$ & $\begin{array}{c}\text { GR-22 } \\
10\end{array}$ \\
\hline \multicolumn{2}{|c|}{$\mathrm{SiO}_{2}$} & 49.48 & 50.37 & 49.91 & 49.34 & 49.38 & 49.38 & 48.86 & 49.09 & 50.09 & 48.57 & 47.88 & 50.34 \\
\hline \multicolumn{2}{|c|}{$\mathrm{TiO}_{2}$} & 1.95 & 2.32 & 1.65 & 2.00 & 2.37 & 1.60 & 1.64 & 1.95 & 2.06 & 1.90 & 1.84 & 2.99 \\
\hline \multicolumn{2}{|c|}{$\mathrm{Al}_{2} \mathrm{O}_{3}$} & 14.13 & 13.87 & 15.25 & 13.64 & 13.84 & 14.32 & 14.72 & 13.90 & 13.30 & 15.61 & 15.72 & 13.70 \\
\hline \multicolumn{2}{|c|}{$\mathrm{Fe}_{2} \mathrm{O}_{3}$} & 1.78 & 1.56 & 1.04 & 3.03 & 1.34 & 2.54 & 3.41 & 2.62 & 3.43 & 1.20 & 1.59 & 2.28 \\
\hline \multicolumn{2}{|c|}{$\mathrm{FeO}$} & 11.26 & 12.79 & 10.00 & 9.94 & 12.65 & 9.96 & 7.78 & 9.59 & 9.19 & 9.92 & 9.69 & 11.64 \\
\hline \multicolumn{2}{|c|}{$\mathrm{MnO}$} & 0.23 & 0.24 & 0.19 & 0.19 & 0.25 & 0.18 & 0.21 & 0.21 & 0.22 & 0.19 & 0.17 & 0.25 \\
\hline \multicolumn{2}{|c|}{ MgO } & 6.50 & 5.60 & 7.80 & 6.41 & 5.86 & 6.94 & 7.27 & 6.78 & 6.80 & 7.79 & 7.97 & 5.34 \\
\hline \multicolumn{2}{|c|}{$\mathrm{CaO}$} & 11.18 & 10.37 & 11.64 & 11.25 & 10.45 & 11.54 & 12.59 & 11.68 & 11.70 & 11.59 & 11.86 & 9.67 \\
\hline \multicolumn{2}{|c|}{$\mathrm{Na}_{2} \mathrm{O}$} & 2.39 & 2.25 & 2.07 & 2.58 & 2.50 & 2.32 & 2.22 & 2.33 & 2.42 & 2.25 & 2.31 & 2.86 \\
\hline \multicolumn{2}{|c|}{$\mathrm{K}_{2} \mathrm{O}$} & 0.29 & 0.32 & 0.24 & 0.28 & 0.29 & 0.23 & 0.21 & 0.27 & 0.26 & 0.23 & 0.23 & 0.48 \\
\hline \multicolumn{2}{|c|}{$\mathrm{P}_{2} \mathrm{O}_{5}$} & 0.23 & 0.28 & 0.17 & 0.25 & 0.23 & 0.15 & 0.19 & 0.22 & 0.23 & 0.19 & 0.18 & 0.33 \\
\hline \multicolumn{2}{|c|}{ Total } & 99.42 & 99.97 & 99.96 & 98.91 & 99.16 & 99.16 & 99.10 & 98.64 & 99.70 & 99.44 & 99.44 & 99.89 \\
\hline \multicolumn{14}{|c|}{ CIPW norms (recalculated to $100 \%$ ) } \\
\hline \multicolumn{2}{|l|}{$\mathrm{Qz}$} & - & 2.64 & - & 0.83 & 0.17 & 0.14 & 0.53 & 0.70 & 2.32 & - & - & 2.17 \\
\hline \multicolumn{2}{|c|}{ Or } & 1.72 & 1.89 & 1.42 & 1.67 & 1.73 & 1.37 & 1.25 & 1.62 & 1.54 & 1.37 & 1.37 & 2.84 \\
\hline \multicolumn{2}{|c|}{$\mathrm{Ab}$} & 20.34 & 19.04 & 17.52 & 22.07 & 21.33 & 19.80 & 18.96 & 19.99 & 20.54 & 19.15 & 19.66 & 24.23 \\
\hline \multicolumn{2}{|c|}{ An } & 27.13 & 26.81 & 31.64 & 25.08 & 25.90 & 28.22 & 29.85 & 27.04 & 24.73 & 31.99 & 32.02 & 23.15 \\
\hline & Wo & 11.34 & 9.53 & 10.45 & 12.40 & 10.38 & 11.91 & 13.33 & 12.63 & 13.35 & 10.26 & 10.84 & 9.48 \\
\hline \multirow[t]{2}{*}{ Di } & En & 5.53 & 4.06 & 5.67 & 6.65 & 4.55 & 6.39 & 8.32 & 6.96 & 7.71 & 5.66 & 6.13 & 4.41 \\
\hline & Fs & 5.61 & 5.48 & 4.42 & 5.33 & 5.82 & 5.13 & 4.20 & 5.20 & 5.03 & 4.21 & 4.25 & 4.97 \\
\hline & En & 10.42 & 9.89 & 12.45 & 9.49 & 10.17 & 11.04 & 9.95 & 10.16 & 9.28 & 8.26 & 5.22 & 8.93 \\
\hline & Fs & 10.57 & 13.33 & 9.70 & 7.60 & 13.01 & 8.87 & 5.03 & 7.59 & 6.05 & 6.14 & 3.62 & 10.06 \\
\hline & Fo & 0.23 & - & 0.92 & - & - & - & - & - & - & 3.92 & 6.03 & - \\
\hline & $\mathrm{Fa}$ & 0.26 & - & 0.79 & - & - & - & - & - & - & 3.21 & 4.61 & - \\
\hline \multicolumn{2}{|l|}{ II } & 3.72 & 4.41 & 3.13 & 3.84 & 4.54 & 3.06 & 3.14 & 3.75 & 3.92 & 3.63 & 3.51 & 5.68 \\
\hline \multicolumn{2}{|c|}{$\mathrm{Mt}$} & 2.60 & 2.26 & 1.51 & 4.44 & 1.96 & 3.71 & 4.99 & 3.85 & 4.99 & 1.75 & 2.32 & 3.31 \\
\hline \multicolumn{2}{|c|}{ Ap } & 0.54 & 0.65 & 0.39 & 0.59 & 0.54 & 0.35 & 0.44 & 0.52 & 0.53 & 0.44 & 0.42 & 0.77 \\
\hline
\end{tabular}


(Table 2 continued).

\begin{tabular}{|c|c|c|c|c|c|c|c|c|c|c|c|c|c|}
\hline \multicolumn{2}{|c|}{$\begin{array}{l}\text { Sample No. } \\
\text { No. of anal. }\end{array}$} & $\begin{array}{c}\text { GR-23A } \\
8\end{array}$ & $\begin{array}{c}\text { GR-23B } \\
5\end{array}$ & $\begin{array}{c}\mathrm{KE}-24 \\
8\end{array}$ & $\begin{array}{c}\mathrm{HE}-30 \\
12\end{array}$ & $\begin{array}{c}\mathrm{HE}-32 \\
10\end{array}$ & $\begin{array}{c}\mathrm{HE}-35 \\
11\end{array}$ & $\begin{array}{c}\text { LA-38 } \\
8\end{array}$ & $\begin{array}{c}\text { TJ-96 } \\
13\end{array}$ & $\begin{array}{c}\text { TJ-99 } \\
6\end{array}$ & $\begin{array}{c}\mathrm{TJ}-101^{\mathrm{a}} \\
14\end{array}$ & $\begin{array}{c}\mathrm{TJ}-101^{\mathrm{b}} \\
11\end{array}$ & $\begin{array}{c}\text { TJ-104 } \\
12\end{array}$ \\
\hline \multicolumn{2}{|c|}{$\mathrm{SiO}_{2}$} & 49.64 & 50.52 & 49.64 & 48.68 & 48.26 & 48.21 & 50.00 & 53.16 & 47.14 & 46.90 & 48.98 & 48.53 \\
\hline \multicolumn{2}{|c|}{$\mathrm{TiO}_{2}$} & 2.95 & 3.02 & 2.88 & 1.25 & 1.21 & 1.11 & 2.15 & 1.09 & 2.61 & 2.47 & 2.15 & 1.08 \\
\hline \multicolumn{2}{|c|}{$\mathrm{Al}_{2} \mathrm{O}_{3}$} & 13.40 & 13.77 & 13.72 & 14.70 & 15.36 & 14.98 & 13.73 & 15.89 & 14.97 & 13.38 & 15.69 & 15.30 \\
\hline \multicolumn{2}{|c|}{$\mathrm{Fe}_{2} \mathrm{O}_{3}$} & 1.86 & 1.94 & 3.22 & 1.39 & 1.35 & 1.37 & 2.55 & 1.11 & 1.64 & 1.52 & 1.71 & 1.31 \\
\hline \multicolumn{2}{|c|}{$\mathrm{FeO}$} & 12.42 & 12.98 & 11.72 & 9.32 & 9.14 & 9.24 & 10.44 & 7.42 & 10.95 & 10.11 & 11.42 & 8.74 \\
\hline \multicolumn{2}{|c|}{$\mathrm{MnO}$} & 0.19 & 0.28 & 0.24 & 0.18 & 0.17 & 0.17 & 0.21 & 0.15 & 0.20 & 0.29 & 0.24 & 0.18 \\
\hline \multicolumn{2}{|c|}{$\mathrm{MgO}$} & 5.57 & 5.59 & 4.94 & 8.53 & 8.73 & 8.56 & 6.22 & 6.34 & 7.18 & 8.75 & 6.43 & 9.21 \\
\hline \multicolumn{2}{|c|}{$\mathrm{CaO}$} & 9.81 & 10.14 & 10.15 & 13.05 & 13.13 & 13.53 & 11.23 & 10.78 & 11.62 & 14.79 & 11.40 & 13.91 \\
\hline \multicolumn{2}{|c|}{$\mathrm{Na}_{2} \mathrm{O}$} & 3.08 & 2.01 & 2.92 & 1.95 & 1.98 & 1.90 & 2.65 & 2.32 & 2.31 & 1.25 & 2.62 & 1.87 \\
\hline \multicolumn{2}{|c|}{$\mathrm{K}_{2} \mathrm{O}$} & 0.48 & 0.47 & 0.55 & 0.09 & 0.10 & 0.08 & 0.34 & 0.59 & 0.21 & 0.08 & 0.20 & 0.03 \\
\hline \multicolumn{2}{|c|}{$\mathrm{P}_{2} \mathrm{O}_{5}$} & 0.31 & 0.33 & 0.35 & 0.11 & 0.10 & 0.10 & 0.26 & 0.10 & 0.21 & 0.26 & 0.08 & 0.06 \\
\hline \multicolumn{2}{|c|}{ Total } & 99.72 & 101.00 & 100.33 & 99.25 & 99.53 & 99.25 & 99.78 & 98.95 & 99.04 & 99.79 & 100.92 & 100.22 \\
\hline \multicolumn{14}{|c|}{ CIPW norms (recalculated to $100 \%$ ) } \\
\hline \multicolumn{2}{|c|}{$\mathrm{Qz}$} & - & 4.34 & 1.27 & - & - & - & 0.71 & 4.77 & - & - & - & - \\
\hline \multicolumn{2}{|l|}{ Or } & 2.84 & 2.75 & 3.24 & 0.54 & 0.59 & 0.48 & 2.01 & 3.52 & 1.25 & 0.47 & 1.17 & 0.18 \\
\hline \multicolumn{2}{|c|}{$\mathrm{Ab}$} & 26.14 & 16.84 & 24.63 & 16.63 & 16.83 & 16.20 & 22.47 & 19.84 & 19.74 & 10.60 & 21.97 & 15.79 \\
\hline \multicolumn{2}{|c|}{ An } & 21.38 & 26.89 & 22.63 & 31.33 & 32.88 & 32.35 & 24.62 & 31.53 & 30.15 & 30.73 & 30.18 & 33.19 \\
\hline \multirow{3}{*}{ Di } & Wo & 10.60 & 8.68 & 10.56 & 13.85 & 13.32 & 14.46 & 12.32 & 9.13 & 11.14 & 17.16 & 10.58 & 14.73 \\
\hline & En & 4.78 & 3.81 & 4.80 & 7.93 & 7.73 & 8.27 & 6.33 & 5.12 & 5.90 & 10.02 & 5.13 & 8.79 \\
\hline & Fs & 5.77 & 4.84 & 5.68 & 5.31 & 4.97 & 5.55 & 5.68 & 3.63 & 4.89 & 6.33 & 5.27 & 5.18 \\
\hline & En & 7.87 & 9.97 & 7.46 & 7.35 & 5.06 & 4.93 & 9.20 & 10.83 & 5.63 & 7.09 & 5.57 & 4.09 \\
\hline & Fs & 9.51 & 12.66 & 8.82 & 4.92 & 3.25 & 3.31 & 8.25 & 7.67 & 4.66 & 4.48 & 5.72 & 2.41 \\
\hline & Fo & 0.89 & - & - & 4.29 & 6.35 & 5.81 & - & - & 4.57 & 3.31 & 3.62 & 7.02 \\
\hline & $\mathrm{Fa}$ & 1.18 & - & 一 & 3.17 & 4.50 & 4.30 & - & 一 & 4.17 & 2.31 & 4.10 & 4.56 \\
\hline \multirow{2}{*}{\multicolumn{2}{|c|}{$\begin{array}{l}\text { Il } \\
\text { Mt }\end{array}$}} & 5.62 & 5.68 & 5.45 & 2.39 & 2.31 & 2.12 & 4.09 & 2.09 & 5.00 & 4.70 & 4.05 & 2.05 \\
\hline & & 2.71 & 2.72 & 4.65 & 2.03 & 1.97 & 2.00 & 3.71 & 1.63 & 2.40 & 2.20 & 2.46 & 1.90 \\
\hline \multicolumn{2}{|c|}{ Ap } & 0.72 & 0.76 & 0.81 & 0.26 & 0.23 & 0.23 & 0.60 & 0.23 & 0.49 & 0.60 & 0.18 & 0.14 \\
\hline
\end{tabular}


Table 3. Microprobe analyses of olivines. ma - macrophenocryst mi - microphenocryst. Sample location is shown on the map (Fig. 5) and given in Appendix 1.

\begin{tabular}{|c|c|c|c|c|c|c|c|c|c|c|c|c|c|c|c|c|}
\hline $\begin{array}{l}\text { Sample No. } \\
\text { No. of anal. }\end{array}$ & $\begin{array}{c}1 \\
15\end{array}$ & $\begin{array}{l}3 \\
7\end{array}$ & $\underset{2}{4 \mathrm{ma}}$ & $\begin{array}{c}4 \mathrm{mi} \\
2\end{array}$ & $\underset{1}{5 \mathrm{ma}}$ & $\underset{7}{5 \mathrm{mi}}$ & $\begin{array}{l}7 \\
3\end{array}$ & $\begin{array}{l}10 \\
11\end{array}$ & $\underset{3}{11 \mathrm{ma}}$ & $\underset{6}{11 \mathrm{mi}}$ & $\begin{array}{l}16 \\
21\end{array}$ & $\begin{array}{c}17 \\
8\end{array}$ & $\begin{array}{c}19 \mathrm{ma} \\
7\end{array}$ & $\begin{array}{c}19 \mathrm{mi} \\
5\end{array}$ & $\begin{array}{c}20 \mathrm{ma} \\
4\end{array}$ & $\begin{array}{c}20 \mathrm{mi} \\
2\end{array}$ \\
\hline $\mathrm{SiO}_{2}$ & 39.40 & 39.26 & 39.11 & 38.86 & 39.51 & 39.37 & 38.66 & 39.10 & 39.74 & 39.47 & 38.94 & 38.52 & 39.16 & 39.00 & 38.65 & 38.76 \\
\hline $\mathrm{FeO}^{\mathrm{t}}$ & 19.75 & 22.62 & 15.97 & 16.32 & 16.52 & 20.17 & 22.43 & 19.15 & 10.97 & 14.62 & 17.69 & 18.97 & 15.05 & 14.87 & 14.81 & 18.40 \\
\hline $\mathrm{MnO}$ & 0.30 & 0.34 & 0.23 & 0.23 & 0.28 & 0.32 & 0.38 & 0.29 & 0.19 & 0.19 & 0.27 & 0.31 & 0.19 & 0.26 & 0.24 & 0.27 \\
\hline $\mathrm{MgO}$ & 40.42 & 38.68 & 43.93 & 45.18 & 42.56 & 39.75 & 39.06 & 40.52 & 48.67 & 46.45 & 42.11 & 43.90 & 43.58 & 43.86 & 46.77 & 44.51 \\
\hline $\mathrm{CaO}$ & 0.33 & 0.30 & 0.33 & 0.33 & 0.23 & 0.34 & 0.35 & 0.36 & 0.37 & 0.37 & 0.34 & 0.32 & 0.29 & 0.33 & 0.32 & 0.45 \\
\hline Total & 100.20 & 101.20 & 99.57 & 100.92 & 99.10 & 99.95 & 100.88 & 99.42 & 99.94 & 101.10 & 99.34 & 102.02 & 98.27 & 98.32 & 100.79 & 102.39 \\
\hline \multicolumn{17}{|c|}{ Numbers of ions on the basis of $4(0)$} \\
\hline $\mathrm{Si}$ & 1.01 & 1.01 & 0.99 & 0.98 & 1.01 & 1.01 & 1.00 & 1.01 & 0.98 & 0.98 & 1.00 & 0.97 & 1.00 & 1.00 & 0.97 & 0.97 \\
\hline $\mathrm{Fe}$ & 0.42 & 0.49 & 0.34 & 0.34 & 0.35 & 0.43 & 0.48 & 0.41 & 0.23 & 0.30 & 0.38 & 0.40 & 0.32 & 0.32 & 0.31 & 0.38 \\
\hline $\mathrm{Mn}$ & 0.01 & 0.01 & 0.00 & 0.00 & 0.01 & 0.01 & 0.01 & 0.01 & 0.00 & 0.00 & 0.01 & 0.01 & 0.00 & 0.01 & 0.01 & 0.01 \\
\hline $\mathrm{Mg}$ & 1.54 & 1.48 & 1.66 & 1.69 & 1.62 & 1.52 & 1.50 & 1.56 & 1.79 & 1.72 & 1.61 & 1.65 & 1.66 & 1.67 & 1.74 & 1.66 \\
\hline $\mathrm{Ca}$ & 0.01 & 0.01 & 0.01 & 0.01 & 0.01 & 0.01 & 0.01 & 0.01 & 0.01 & 0.01 & 0.01 & 0.01 & 0.01 & 0.01 & 0.01 & 0.01 \\
\hline Fo $($ mole- $\%$ o) & 78.49 & 75.29 & 83.06 & 83.14 & 82.11 & 77.62 & 75.63 & 79.04 & 88.77 & 84.99 & 80.91 & 80.49 & 83.77 & 84.01 & 84.91 & 81.17 \\
\hline
\end{tabular}

(Table 3 continued).

\begin{tabular}{|c|c|c|c|c|c|c|c|c|c|c|c|c|c|c|c|}
\hline $\begin{array}{l}\text { Sample No. } \\
\text { No. of anal. }\end{array}$ & $\begin{array}{c}22 \\
5\end{array}$ & $\begin{array}{c}24 \\
2\end{array}$ & $\begin{array}{c}30 \mathrm{ma} \\
3\end{array}$ & $\begin{array}{c}30 \mathrm{mi} \\
11\end{array}$ & $\begin{array}{c}32 \mathrm{ma} \\
\mathbf{5}\end{array}$ & $\begin{array}{c}32 \mathrm{mi} \\
9\end{array}$ & $\begin{array}{c}35 \\
4\end{array}$ & $\begin{array}{c}38 \\
4\end{array}$ & $\begin{array}{l}96 \\
11\end{array}$ & $\begin{array}{c}99 \mathrm{ma} \\
7\end{array}$ & $\begin{array}{c}99 \mathrm{mi} \\
14\end{array}$ & $\begin{array}{c}101 \mathrm{~A} \\
6\end{array}$ & $\begin{array}{c}101 \mathrm{~B} \\
4\end{array}$ & $\begin{array}{c}104 \mathrm{ma} \\
12\end{array}$ & $\begin{array}{c}104 \mathrm{mi} \\
11\end{array}$ \\
\hline $\mathrm{SiO}_{2}$ & 37.50 & 38.22 & 39.71 & 39.98 & 40.02 & 40.13 & 39.07 & 38.18 & 41.26 & 39.97 & 39.60 & 39.99 & 40.07 & 39.68 & 39.36 \\
\hline $\mathrm{FeO}^{\mathrm{t}}$ & 26.02 & 29.39 & 13.56 & 13.67 & 13.31 & 13.19 & 13.71 & 20.88 & 13.14 & 17.46 & 17.63 & 17.85 & 17.28 & 13.80 & 13.98 \\
\hline $\mathrm{MnO}$ & 0.38 & 0.48 & 0.22 & 0.20 & 0.19 & 0.19 & 0.18 & 0.36 & 0.25 & 0.26 & 0.25 & 0.28 & 0.21 & 0.22 & 0.21 \\
\hline $\mathrm{MgO}$ & 33.12 & 30.62 & 45.72 & 45.52 & 45.92 & 45.12 & 44.53 & 38.88 & 43.42 & 41.60 & 40.47 & 42.86 & 43.42 & 45.43 & 45.82 \\
\hline $\mathrm{CaO}$ & 0.32 & 0.36 & 0.35 & 0.37 & 0.38 & 0.34 & 0.43 & 0.34 & 0.31 & 0.32 & 0.33 & 0.33 & 0.37 & 0.38 & 0.43 \\
\hline Total & 97.34 & 99.07 & 99.56 & 99.74 & 99.82 & 98.97 & 97.92 & 98.64 & 98.65 & 99.61 & 98.28 & 101.31 & 101.35 & 99.51 & 99.80 \\
\hline \multicolumn{16}{|c|}{ Numbers of ions on the basis of $4(0)$} \\
\hline $\mathrm{Si}$ & 1.02 & 1.04 & 1.00 & 1.00 & 1.01 & 1.01 & 1.00 & 1.00 & 1.00 & 1.02 & 1.02 & 1.00 & 1.00 & 1.00 & 0.99 \\
\hline $\mathrm{Fe}$ & 0.59 & 0.67 & 0.28 & 0.29 & 0.28 & 0.28 & 0.29 & 0.46 & 0.39 & 0.37 & 0.38 & 0.37 & 0.36 & 0.29 & 0.29 \\
\hline $\mathrm{Mn}$ & 0.01 & 0.01 & 0.00 & 0.00 & 0.00 & 0.00 & 0.00 & 0.01 & 0.01 & 0.01 & 0.01 & 0.01 & 0.00 & 0.00 & 0.00 \\
\hline $\mathrm{Mg}$ & 1.35 & 1.24 & 1.71 & 1.70 & 1.71 & 1.69 & 1.70 & 1.52 & 1.59 & 1.58 & 1.56 & 1.60 & 1.62 & 1.70 & 1.71 \\
\hline $\mathrm{Ca}$ & 0.01 & 0.01 & 0.01 & 0.01 & 0.01 & 0.01 & 0.01 & 0.01 & 0.01 & 0.01 & 0.01 & 0.01 & 0.01 & 0.01 & 0.01 \\
\hline Fo $(\mathrm{mole} \% \%)$ & 69.40 & 64.99 & 85.73 & 85.58 & 86.01 & 85.91 & 85.23 & 76.84 & 85.23 & 80.94 & 80.36 & 81.06 & 81.75 & 85.44 & 85.38 \\
\hline
\end{tabular}


Fig. 9. Frequency histogram of $\mathrm{CaO}$ content in olivine. Each block equals one analysis. The separation between low $\mathrm{Ca}$ olivines of plutonic rocks and high $\mathrm{Ca}$ olivines of extrusive and hypabyssal rocks is shown.

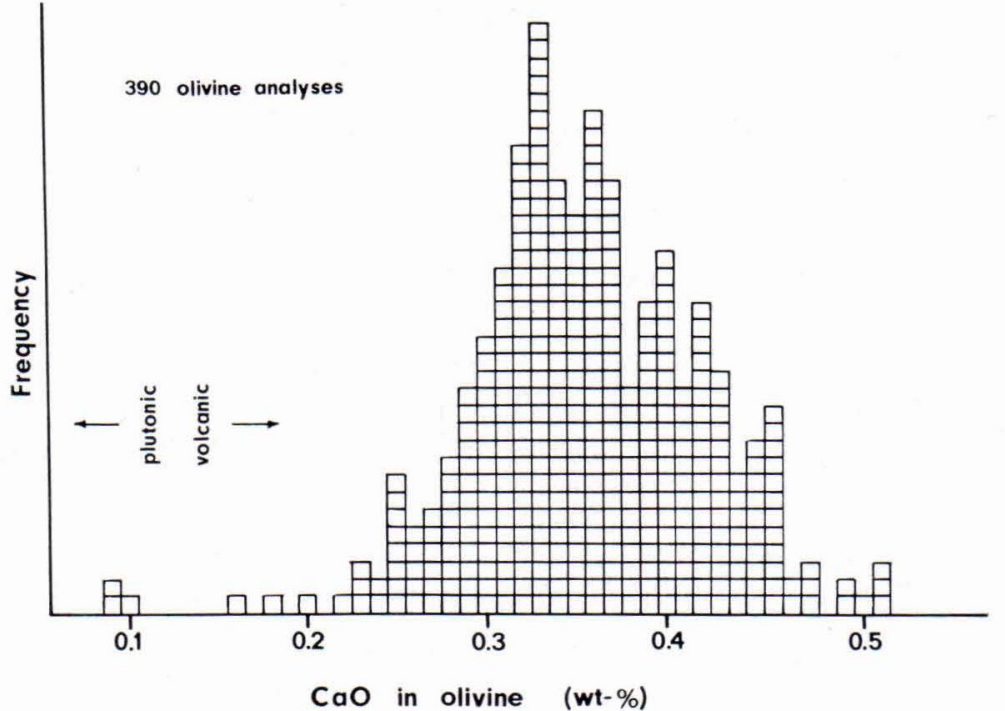

All the analyzed plagioclase crystals have relatively high but variable contents of $\mathrm{FeO}$ and $\mathrm{MgO}$. FeO content is higher in microphenocrysts than in macrophenocrysts. Hafner et al. (1971) and Quaide (1972) suggested that $\mathrm{Fe}$ and $\mathrm{Mg}$ do not enter the plagioclase lattice in lunar plagioclases. They concluded that iron especially exists mainly at irregular structural sites. Therefore the $\mathrm{Fe}+\mathrm{Mg}$ and $\mathrm{Fe} /(\mathrm{Fe}+\mathrm{Mg})$ should reflect the coexisting magma.

Bryan and Kullerud (1969) and Taylor et al. (1971) suggested, however, that Fe probably substitutes for $\mathrm{Al}$ in lunar plagioclases and that divalent $\mathrm{Ca}, \mathrm{Mg}$ and $\mathrm{Fe}$ can form the unit of the type $\mathrm{Ca}(\mathrm{Mg}, \mathrm{Fe}) \mathrm{Si}_{3} \mathrm{O}_{8}$.

All the analyzed plagioclases show strong positive correlation of $\mathrm{FeO}+\mathrm{MgO}$ with albite content. This correlation is significantly different for quartz normative tholeiites and olivine normative tholeiites. When the albite content ranges from 17 to 42 (mole- $\%$ ) the $\mathrm{FeO}+\mathrm{MgO}$ content changes from 0.84 to 1.30 in quartz normative tholeiites. In olivine normative tholeiites the corresponding values are $\mathrm{Ab} 13-28 \quad\left(\mathrm{~mole}^{-} \%\right)$ and $\mathrm{FeO}+\mathrm{MgO}$ $0.65-1.35$.
The phenocrysts show a large variation of crystal morphology (Fig. 10). Microphenocrysts are usually small laths (acicular), usually only $5 \mu \mathrm{m}$ broad and $20-40 \mu \mathrm{m}$ long. They may also be skeletal or hollow. According to Löfgren (1974) this morphological type is what can be expected in basalts of subaquatic origin. Tabular morphology is observed too.

The macrophenocrysts are large up to 3 $\mathrm{mm}$. They are frequently rounded but large euhedral plagioclases are present as well. In some samples crystal fragments occur. Some corroded plagioclases observed indicate that they are probably xenocrysts.

Clinopyroxene. Clinopyroxene is present in most of the samples. Usually they are poorly developed in the basalts studied. Microphenocrysts are more common than macrophenocrysts. The plotting of partial microprobe analyses (Table 5) on the $\mathrm{Mg}-\mathrm{Ca}-\mathrm{Fe}$ diagram (Fig. 11) shows that all clinopyroxenes fall very near the Skaegaard fractionation trend. Carmichael (1967) reported phenocrysts and groundmass clinopyroxene trends which are also shown in Fig. 11. 

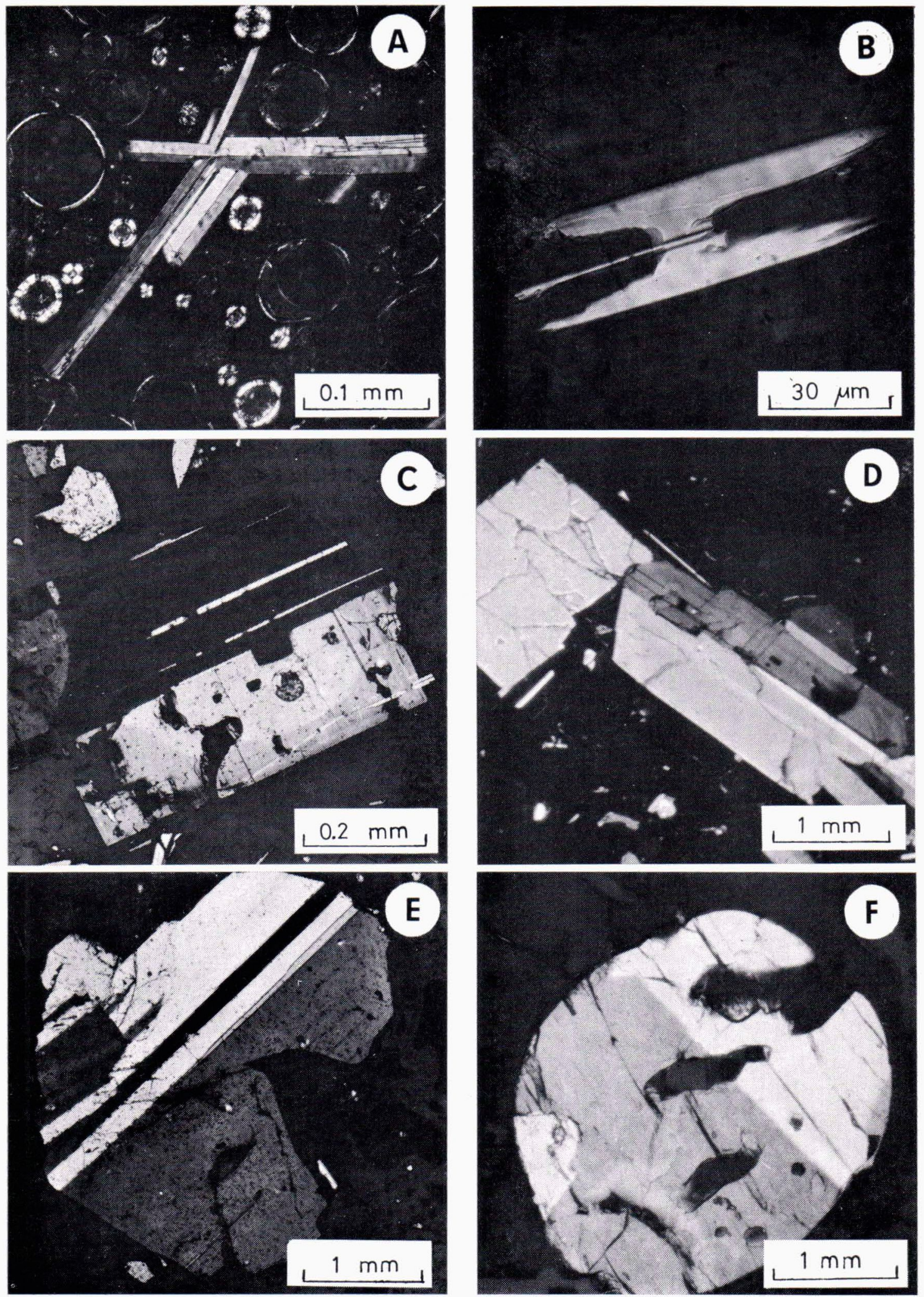

Fig. 10. Plagioclase morphologies. A euhedral (acicular) plagioclase microphenocrysts (RE-11), B shallow-tailed plagioclase (RE-19), C normally zoned broken plagioclase phenocryst (RE-17), D euhedral plagioclase macrophenocryst (RE-19), E skeletal plagioclase (tabular shaped) (RE-17), F anhedral plagioclase xenolith (LA-38) 
Table 4. Microprobe analyses of plagioclases. ma - macrophenocryst, mi - microphenocryst. Sample location is shown on the map (Fig. 5) and given in Appendix 1.

\begin{tabular}{|c|c|c|c|c|c|c|c|c|c|c|c|c|c|c|}
\hline $\begin{array}{l}\text { Sample No. } \\
\text { No. of anal. }\end{array}$ & $1 \mathrm{ma}$ & $\underset{8}{1 \mathrm{mi}}$ & $\begin{array}{c}3 \mathrm{ma} \\
2\end{array}$ & $\begin{array}{c}3 \mathrm{mi} \\
11\end{array}$ & $\begin{array}{c}4 \mathrm{ma} \\
2\end{array}$ & $\begin{array}{c}4 \mathrm{mi} \\
8\end{array}$ & $\begin{array}{c}5 \mathrm{ma} \\
1\end{array}$ & $\begin{array}{c}5 \mathrm{mi} \\
7\end{array}$ & $\begin{array}{c}7 \\
10\end{array}$ & $\begin{array}{c}10 \mathrm{ma} \\
1\end{array}$ & $\begin{array}{c}10 \mathrm{mi} \\
9\end{array}$ & $\underset{2}{11 \mathrm{ma}}$ & $\underset{4}{11 \mathrm{mi}}$ & $\begin{array}{c}16 \mathrm{ma} \\
9\end{array}$ \\
\hline $\mathrm{SiO}_{2}$ & 48.74 & 52.32 & 53.05 & 53.75 & 48.69 & 48.59 & 47.61 & 51.51 & 51.32 & 47.40 & 50.76 & 50.49 & 49.77 & 48.00 \\
\hline $\mathrm{Al}_{2} \mathrm{O}_{3}$ & 33.99 & 29.98 & 30.40 & 30.09 & 34.82 & 34.69 & 32.99 & 29.27 & 30.59 & 33.57 & 29.88 & 33.40 & 33.18 & 33.22 \\
\hline $\mathrm{FeO}^{\mathrm{t}}$ & 0.64 & 0.94 & 0.79 & 0.99 & 0.43 & 0.46 & 0.64 & 0.79 & 0.90 & 0.38 & 0.74 & 0.64 & 0.65 & 0.66 \\
\hline MgO & 0.21 & 0.41 & 0.24 & 0.26 & 0.19 & 0.19 & 0.21 & 0.19 & 0.22 & 0.16 & 0.31 & 0.29 & 0.27 & 0.18 \\
\hline $\mathrm{CaO}$ & 16.87 & 13.61 & 12.73 & 11.87 & 17.45 & 16.99 & 16.27 & 13.52 & 13.39 & 17.12 & 13.73 & 16.20 & 16.22 & 16.56 \\
\hline $\mathrm{Na}_{2} \mathrm{O}$ & 1.86 & 3.06 & 3.81 & 3.80 & 1.46 & 1.45 & 1.89 & 3.30 & 4.08 & 1.76 & 3.40 & 2.55 & 2.57 & 1.90 \\
\hline $\mathrm{K}_{2} \mathrm{O}$ & 0.02 & 0.07 & 0.07 & 0.08 & 0.02 & 0.01 & 0.01 & 0.07 & 0.07 & 0.01 & 0.06 & 0.03 & 0.02 & 0.00 \\
\hline Total & 102.33 & 100.39 & 101.09 & 100.84 & 103.04 & 102.38 & 99.65 & 98.65 & 100.57 & 100.40 & 98.88 & 103.60 & 102.68 & 100.52 \\
\hline \multicolumn{15}{|c|}{ Numbers of ions on the basis of $32(0)$} \\
\hline $\mathrm{Si}$ & 8.75 & 9.48 & 9.52 & 9.64 & 8.67 & 8.70 & 8.77 & 9.50 & 9.32 & 8.68 & 9.36 & 8.93 & 8.90 & 8.77 \\
\hline $\mathrm{Al}$ & 7.19 & 6.40 & 6.43 & 6.36 & 7.31 & 7.32 & 7.16 & 6.37 & 6.55 & 7.25 & 6.50 & 6.97 & 6.99 & 7.15 \\
\hline $\mathrm{Fe}$ & 0.10 & 0.14 & 0.12 & 0.15 & 0.06 & 0.07 & 0.10 & 0.12 & 0.14 & 0.06 & 0.11 & 0.09 & 0.10 & 0.10 \\
\hline Mg & 0.06 & 0.11 & 0.06 & 0.07 & 0.05 & 0.05 & 0.06 & 0.05 & 0.06 & 0.04 & 0.09 & 0.08 & 0.07 & 0.05 \\
\hline $\mathrm{Ca}$ & 3.24 & 2.64 & 2.45 & 2.28 & 3.33 & 3.26 & 3.21 & 2.67 & 2.61 & 3.36 & 2.71 & 3.07 & 3.11 & 3.24 \\
\hline $\mathrm{Na}$ & 0.65 & 1.07 & 1.33 & 1.32 & 0.50 & 0.50 & 0.68 & 1.18 & 1.41 & 0.62 & 1.22 & 0.87 & 0.89 & 0.67 \\
\hline $\mathrm{K}$ & 0.00 & 0.02 & 0.02 & 0.02 & 0.00 & 0.00 & 0.00 & 0.02 & 0.02 & 0.00 & 0.01 & 0.01 & 0.00 & 0.00 \\
\hline An $($ mole- $\%)$ & 83.50 & 71.61 & 65.18 & 63.69 & 86.91 & 86.74 & 82.72 & 69.32 & 64.57 & 84.43 & 69.46 & 78.12 & 78.02 & 83.01 \\
\hline
\end{tabular}

(Table 4 continued, 1).

\begin{tabular}{|c|c|c|c|c|c|c|c|c|c|c|c|c|c|c|}
\hline $\begin{array}{l}\text { Sample No. } \\
\text { No of anal. }\end{array}$ & $\underset{5}{16 \mathrm{mi}}$ & $\underset{2}{17 \mathrm{ma}}$ & $\begin{array}{l}17 \mathrm{mi} \\
4\end{array}$ & $\underset{4}{19 \mathrm{ma}}$ & $\begin{array}{c}19 \mathrm{mi} \\
11\end{array}$ & $\begin{array}{c}20 \\
7\end{array}$ & $\begin{array}{c}22 \mathrm{ma} \\
3\end{array}$ & $\begin{array}{c}22 \mathrm{mi} \\
9\end{array}$ & $\underset{1}{24 \mathrm{ma}}$ & $\begin{array}{c}24 \mathrm{mi} \\
8\end{array}$ & $\begin{array}{c}30 \mathrm{ma} \\
6\end{array}$ & $\begin{array}{c}30 \mathrm{mi} \\
4\end{array}$ & $\begin{array}{c}32 \\
6\end{array}$ & $\begin{array}{l}35 \\
10\end{array}$ \\
\hline $\mathrm{SiO}_{2}$ & 50.16 & 50.90 & 48.74 & 49.02 & 48.57 & 49.62 & 48.88 & 50.31 & 56.64 & 54.51 & 50.39 & 48.49 & 49.00 & 48.32 \\
\hline $\mathrm{Al}_{2} \mathrm{O}_{3}$ & 30.98 & 30.49 & 30.57 & 31.75 & 32.17 & 31.21 & 32.62 & 31.03 & 28.34 & 28.86 & 32.04 & 31.74 & 32.63 & 32.28 \\
\hline $\mathrm{FeO}$ & 0.70 & 0.39 & 0.69 & 0.69 & 0.73 & 0.76 & 0.73 & 0.96 & 0.67 & 0.97 & 0.66 & 0.67 & 0.52 & 0.52 \\
\hline $\mathrm{MgO}$ & 0.30 & 0.32 & 0.30 & 0.22 & 0.19 & 0.21 & 0.22 & 0.21 & 0.20 & 0.23 & 0.35 & 0.29 & 0.33 & 0.31 \\
\hline $\mathrm{CaO}$ & 14.95 & 13.72 & 14.68 & 15.22 & 15.47 & 15.10 & 16.37 & 13.96 & 10.69 & 11.89 & 14.71 & 16.53 & 16.22 & 16.71 \\
\hline $\mathrm{Na}{ }_{2} \mathrm{O}$ & 2.91 & 3.28 & 3.09 & 3.19 & 2.81 & 3.10 & 2.19 & 3.83 & 5.32 & 4.77 & 2.39 & 2.31 & 2.35 & 2.31 \\
\hline $\mathrm{K}_{2} \mathrm{O}$ & 0.05 & 0.07 & 0.03 & 0.03 & 0.00 & 0.05 & 0.05 & 0.09 & 0.18 & 0.14 & 0.00 & 0.02 & 0.03 & 0.02 \\
\hline Total & 100.05 & 99.17 & 98.10 & 100.12 & 99.94 & 100.05 & 101.06 & 100.39 & 102.04 & 101.37 & 100.00 & 100.05 & 101.08 & 100.47 \\
\hline \multicolumn{15}{|c|}{ Numbers of ions on the basis of $32(0)$} \\
\hline $\mathrm{Si}$ & 9.17 & 9.34 & 9.11 & 8.99 & 8.92 & 9.09 & 8.88 & 9.18 & 10.02 & 9.76 & 9.13 & 8.91 & 8.89 & 8.85 \\
\hline Al & 6.68 & 6.59 & 6.73 & 6.86 & 6.97 & 6.74 & 6.99 & 6.68 & 5.91 & 6.09 & 6.84 & 6.88 & 6.98 & 6.97 \\
\hline $\mathrm{Fe}$ & 0.11 & 0.06 & 0.11 & 0.11 & 0.11 & 0.12 & 0.11 & 0.15 & 0.10 & 0.15 & 0.10 & 0.10 & 0.08 & 0.08 \\
\hline $\mathrm{Mg}$ & 0.08 & 0.09 & 0.08 & 0.06 & 0.05 & 0.06 & 0.06 & 0.06 & 0.05 & 0.06 & 0.09 & 0.08 & 0.09 & 0.08 \\
\hline $\mathrm{Ca}$ & 2.93 & 2.70 & 2.94 & 2.99 & 3.04 & 2.97 & 3.19 & 2.73 & 2.03 & 2.28 & 2.86 & 3.26 & 3.15 & 3.28 \\
\hline $\mathrm{Na}$ & 1.03 & 1.17 & 1.12 & 1.13 & 1.00 & 1.10 & 0.77 & 1.36 & 1.82 & 1.66 & 0.84 & 0.82 & 0.83 & 0.82 \\
\hline $\mathrm{K}$ & 0.01 & 0.02 & 0.01 & 0.01 & 0.00 & 0.01 & 0.01 & 0.02 & 0.04 & 0.03 & 0.00 & 0.00 & 0.01 & 0.00 \\
\hline An $(\mathrm{mole}-\%$ ) & 74.26 & 70.17 & 72.84 & 72.59 & 75.47 & 72.90 & 80.44 & 66.65 & 52.45 & 57.89 & 77.84 & 80.11 & 79.54 & 80.31 \\
\hline
\end{tabular}


(Table 4 continued, 2).

\begin{tabular}{|c|c|c|c|c|c|c|c|c|c|}
\hline $\begin{array}{l}\text { Sample No. } \\
\text { No. of anal. }\end{array}$ & $\begin{array}{c}38 \\
6\end{array}$ & $\begin{array}{c}96 \mathrm{ma} \\
2\end{array}$ & $\begin{array}{c}96 \mathrm{mi} \\
7\end{array}$ & $\begin{array}{c}99 \mathrm{ma} \\
3\end{array}$ & $\begin{array}{l}99 \mathrm{mi} \\
9\end{array}$ & $\begin{array}{c}101 \mathrm{~A} \\
5\end{array}$ & $\begin{array}{c}101 \mathrm{~B} \\
2\end{array}$ & $\begin{array}{c}104 \mathrm{ma} \\
4\end{array}$ & $\begin{array}{c}104 \mathrm{mi} \\
12\end{array}$ \\
\hline $\mathrm{SiO}_{2}$ & 52.03 & 56.86 & 50.21 & 59.37 & 47.98 & 49.94 & 50.13 & 46.44 & 45.66 \\
\hline $\mathrm{Al}_{2} \mathrm{O}_{3}$ & 30.27 & 27.06 & 29.86 & 26.16 & 32.69 & 30.98 & 30.72 & 34.36 & 34.80 \\
\hline $\mathrm{FeO}$ & 0.83 & 0.46 & 1.08 & 0.33 & 0.86 & 1.14 & 0.87 & 0.50 & 0.57 \\
\hline $\mathrm{MgO}$ & 0.32 & 0.05 & 0.55 & 0.43 & 0.23 & 0.32 & 0.31 & 0.24 & 0.21 \\
\hline $\mathrm{CaO}$ & 13.28 & 8.90 & 14.49 & 7.47 & 15.97 & 15.34 & 15.58 & 17.43 & 17.60 \\
\hline $\mathrm{Na}_{2} \mathrm{O}$ & 3.90 & 5.98 & 2.86 & 7.18 & 2.59 & 3.16 & 2.64 & 1.70 & 1.51 \\
\hline $\mathrm{K}_{2} \mathrm{O}$ & 0.11 & 0.16 & 0.07 & 0.30 & 0.03 & 0.09 & 0.05 & 0.01 & 0.02 \\
\hline Total & 100.74 & 99.47 & 99.12 & 100.81 & 100.12 & 100.96 & 100.20 & 100.44 & 100.14 \\
\hline \multicolumn{10}{|c|}{ Numbers of ions on the basis of $32(0)$} \\
\hline $\mathrm{Si}$ & 9.42 & 10.25 & 9.28 & 10.53 & 8.82 & 9.10 & 9.17 & 8.52 & 8.41 \\
\hline $\mathrm{Al}$ & 6.46 & 5.75 & 6.50 & 5.47 & 7.08 & 6.65 & 6.62 & 7.43 & $\begin{array}{l}0.41 \\
7.56\end{array}$ \\
\hline $\mathrm{Fe}$ & 0.13 & 0.07 & 0.17 & 0.05 & 0.13 & 0.17 & 0.13 & 0.08 & 0.09 \\
\hline $\mathrm{Mg}$ & 0.09 & 0.01 & 0.15 & 0.13 & 0.11 & 0.08 & 0.06 & 0.06 & 0.07 \\
\hline $\mathrm{Ca}$ & 2.58 & 1.72 & 2.87 & 1.42 & 3.14 & 2.99 & 3.05 & 3.43 & 3.48 \\
\hline $\mathrm{Na}$ & 1.37 & 2.08 & 1.02 & 2.47 & 0.92 & 1.12 & 0.94 & 0.60 & 0.54 \\
\hline $\mathrm{K}$ & 0.03 & 0.04 & 0.02 & 0.07 & 0.01 & 0.02 & 0.01 & 0.00 & 0.00 \\
\hline An $($ mole- $\%$ o $)$ & 65.39 & 44.90 & 74.40 & 35.90 & 77.22 & 73.04 & 76.62 & 84.18 & 86.63 \\
\hline
\end{tabular}

The $\mathrm{CaO}$ content of clinopyroxenes increases with decreasing temperature (Green and Ringwood 1967). However, on the basis of the present data the $\mathrm{Ca} / \mathrm{Mg}$ and $\mathrm{Al}_{2} \mathrm{O}_{3}$ variations seem to be more sensitive to the -whole rock composition. Therefore especially Ca-Tschermacitic molecule $\left(\mathrm{CaAl}_{2} \mathrm{SiO}_{6}\right)$ would reflect the whole rock composition and those conditions where fractional crystallization or partial melting took place (Wood 1975).
Microscopic identification of the small pyroxenes in quenched glasses is difficult, because the optical features of pyroxenes and olivines are almost identical. The shape of pyroxene crystals usually differs from olivines but in the rocks studied pyroxenes show similar skeletal and subhedral forms as the olivines. Occasionally pyroxenes are euhedral and twinned making the identification more easy (Fig. 12).

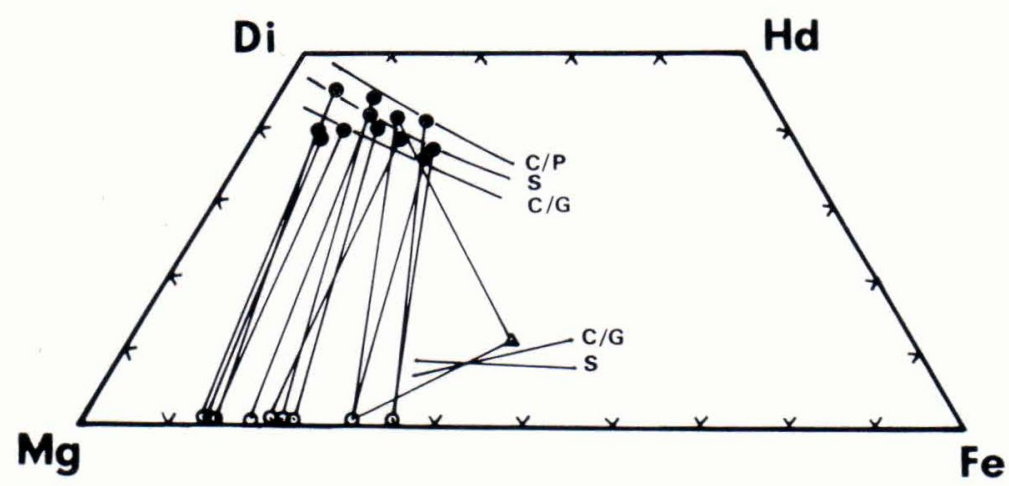

Fig. 11. Average analyses of pyroxenes plotted in terms of $\mathrm{Ca}, \mathrm{Mg}$ and $\mathrm{Fe}+\mathrm{Mn}$.

clinopyroxene, $\mathrm{O}$ - olivine, $\triangle$ - pigeonite. The tie lines indicate mineral assemblages. Curves: S - Skaergaard trend (Deer et al. 1963) C/P Thingmuli phenocryst trend (Charmichael 1967) C/G Thingmuli groundmass trend (Carmichael 1967) 
Table 5. Microprobe analyses of clinopyroxenes. ma - macrophenocryst, mi - microphenocryst, $-=$ not determined, Ca-Ts $=$ Ca-Tschermacitic molecule. Sample location is shown on the map (Fig. 5) and given in Aappendix 1.

\begin{tabular}{|c|c|c|c|c|c|c|c|c|c|c|c|c|}
\hline Sample No. & 1 & 5 & $11 \mathrm{mi}$ & $11 \mathrm{ma}$ & 17 & $22 \mathrm{mi}$ & $22 \mathrm{ma}$ & $24 \mathrm{mi}$ & $24 \mathrm{mi}$ & 30 & 35 & 38 \\
\hline No. of anal. & 1 & 1 & 2 & 1 & 5 & 1 & 6 & 1 & 4 & 2 & 1 & 4 \\
\hline $\mathrm{SiO}_{2}$ & 49.69 & 53.58 & 51.16 & 50.08 & 49.75 & 51.81 & 51.06 & 47.62 & 48.03 & 51.80 & 49.06 & 50.43 \\
\hline $\mathrm{Al}_{2} \mathrm{O}_{3}$ & 4.13 & 4.01 & 2.49 & 5.02 & 4.41 & 2.02 & 1.84 & 4.12 & 4.84 & 6.11 & 5.01 & 4.73 \\
\hline $\mathrm{TiO}_{2}$ & 1.23 & 1.12 & 0.40 & 0.26 & 1.00 & 0.85 & 0.98 & 1.11 & 1.20 & 0.17 & 0.16 & 1.28 \\
\hline $\mathrm{FeO}^{\mathrm{t}}$ & 10.13 & 6.37 & 6.19 & 3.79 & 7.27 & 8.78 & 12.32 & 13.22 & 11.21 & 4.36 & 4.57 & 8.09 \\
\hline $\mathrm{MnO}$ & 0.27 & 0.10 & 0.13 & 0.12 & 0.16 & 0.16 & 0.32 & 0.26 & 0.24 & 0.22 & 0.10 & 0.23 \\
\hline $\mathrm{MgO}$ & 15.31 & 15.45 & 17.98 & 16.85 & 16.01 & 14.85 & 14.30 & 14.98 & 14.00 & 18.45 & 18.57 & 16.46 \\
\hline $\mathrm{CaO}$ & 18.28 & 20.03 & 19.37 & 21.50 & 20.01 & 19.71 & 16.33 & 18.15 & 19.67 & 18.93 & 18.54 & 19.93 \\
\hline $\mathrm{Na} 2 \mathrm{O}$ & 0.28 & 0.25 & 0.18 & 0.18 & 0.28 & 0.30 & 0.28 & 0.37 & 0.48 & 0.27 & 0.00 & 0.35 \\
\hline $\mathrm{Cr}_{2} \mathrm{O}_{3}$ & 0.18 & 0.18 & 0.43 & 0.85 & 0.69 & 0.15 & - & - & - & 0.17 & 0.65 & - \\
\hline Total & 99.50 & 101.09 & 98.33 & 98.65 & 99.58 & 98.63 & 97.43 & 99.83 & 99.67 & 100.48 & 96.66 & 101.45 \\
\hline \multicolumn{13}{|c|}{ Numbers of ions on the basis of $6(0)$} \\
\hline $\mathrm{Si}$ & 1.86 & 1.93 & 1.91 & 1.85 & 1.85 & 1.95 & 1.96 & 1.81 & 1.82 & 1.86 & 1.85 & 1.84 \\
\hline $\mathrm{Al}$ & 0.18 & 0.17 & 0.11 & 0.22 & 0.19 & 0.09 & 0.08 & 0.18 & 0.22 & 0.26 & 0.22 & 0.20 \\
\hline $\mathrm{Ti}$ & 0.03 & 0.03 & 0.01 & 0.01 & 0.03 & 0.02 & 0.03 & 0.03 & 0.03 & 0.00 & 0.00 & 0.04 \\
\hline $\mathrm{Fe}$ & 0.32 & 0.19 & 0.19 & 0.12 & 0.23 & 0.28 & 0.40 & 0.42 & 0.36 & 0.13 & 0.14 & 0.25 \\
\hline Mn & 0.01 & 0.00 & 0.00 & 0.00 & 0.01 & 0.01 & 0.01 & 0.01 & 0.01 & 0.01 & 0.00 & 0.01 \\
\hline $\mathrm{Mg}$ & 0.86 & 0.83 & 1.00 & 0.93 & 0.89 & 0.83 & 0.82 & 0.85 & 0.79 & 0.99 & 1.04 & 0.90 \\
\hline $\mathrm{Ca}$ & 0.73 & 0.77 & 0.77 & 0.85 & 0.80 & 0.79 & 0.67 & 0.74 & 0.80 & 0.73 & 0.75 & 0.78 \\
\hline $\mathrm{Na}$ & 0.02 & 0.02 & 0.01 & 0.01 & 0.02 & 0.02 & 0.02 & 0.03 & 0.04 & 0.02 & 0.00 & 0.02 \\
\hline $\mathrm{Cr}$ & 0.01 & 0.01 & 0.01 & 0.02 & 0.02 & 0.01 & - & - & - & 0.00 & 0.02 & - \\
\hline $100 \times \mathrm{Ca}-\mathrm{Ts}$ & 344.1 & 440.3 & 49.8 & 618.8 & 294.2 & 75.7 & 29.2 & 0.0 & 71.9 & 1185.6 & 538.8 & 292.8 \\
\hline
\end{tabular}




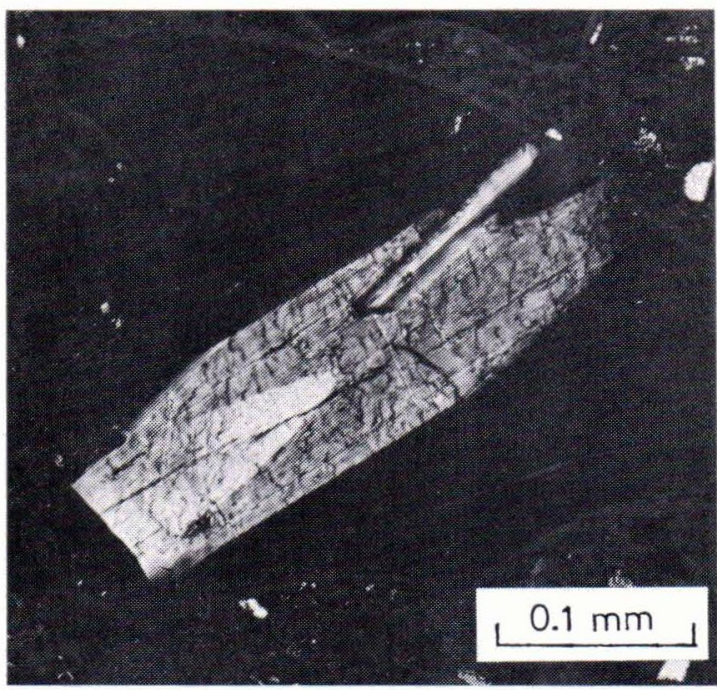

Fig. 12. Hour-glass twinned clinopyroxene (RE-17).

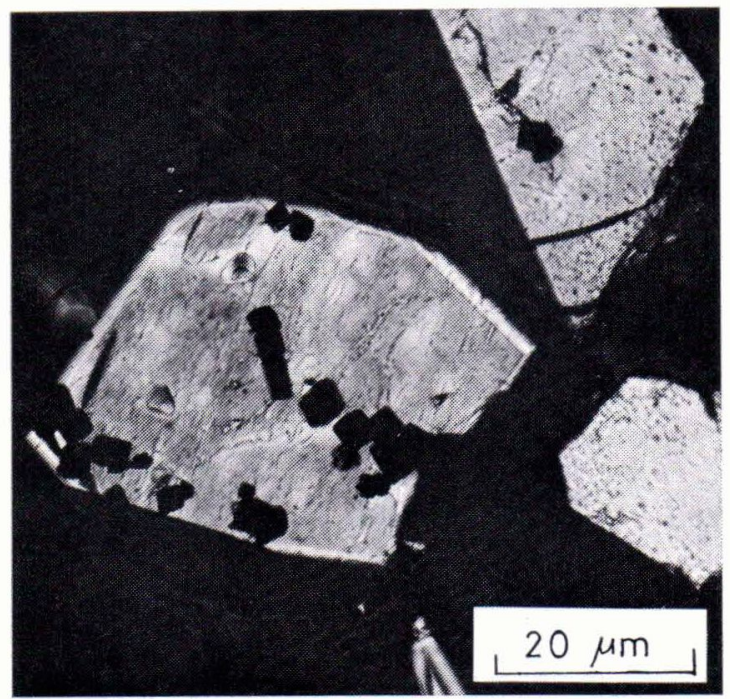

Fig. 13. Chromium-spinel inclusions in olivine (RE-20).

Spinel. Spinel is a common mineral in the rocks studied. Spinel crystals are usually only few $\mu \mathrm{m}(<5 \mu \mathrm{m})$ in diameter. Usually spinel occurs in octahedral grains and forms tiny microphenocrysts which are present as inclusions in olivine and rarely in plagioclase crystals (Fig. 13). They may also be present in the glass as microphenocrysts.
Table 6. Microprobe analyses of spinels. $-=$ not determined. Sample location is shown on the map (Fig. 5) and given in Appendix 1.

\begin{tabular}{|lrrr|}
\hline Sample No. & 11 & $30 \mathrm{~A}$ & $30 \mathrm{~B}$ \\
\hline & & & \\
$\mathrm{SiO}_{2}$ & 1.59 & 0.15 & 0.62 \\
$\mathrm{Al}_{2} \mathrm{O}_{3}$ & 23.34 & 32.09 & 29.54 \\
$\mathrm{TiO}_{2}$ & 1.26 & 0.45 & 0.32 \\
$\mathrm{FeO}$ & 25.54 & 19.40 & 20.66 \\
$\mathrm{MnO}$ & 0.20 & 0.23 & 0.22 \\
$\mathrm{MgO}$ & 12.61 & 15.38 & 16.07 \\
$\mathrm{CaO}$ & 1.32 & 0.02 & - \\
$\mathrm{Cr}$ & 28.89 & 29.97 & 30.50 \\
$\mathrm{NiO}$ & 0.19 & 0.22 & 0.12 \\
$\mathrm{~V}_{2} \mathrm{O}_{3}$ & - & 0.16 & 0.26 \\
$\mathrm{Total}$ & 94.94 & 98.07 & 98.31 \\
& & & \\
\hline
\end{tabular}

Analyses of some typical spinels are given in Table 6, which shows that they are chromium spinels. The low totals result from some difficulties in analyzing the very small grains because of the tendency of the beam to excite adjacent minerals.

Irvine (1967) suggested that high $\mathrm{Al}_{2} \mathrm{O}_{3}$ indicates relatively high pressure of crystallization. However, on the basis of the present limited data no such conclusion is warranted. In the spinels analyzed $\mathrm{Cr}_{2} \mathrm{O}_{3}$ is nearly constant, but $\mathrm{Mg} / \mathrm{Fe}^{t}$ ratio differs more than the analytical error. This $\mathrm{Mg} / \mathrm{Fe}^{\mathrm{t}}$ ratio is higher in the early magmatic chromites indicating that the liquid composition can cause these differences.

The present data has similar tendency in totals as described by Bryan (1971), in other words, the lower the total the lower the $\mathrm{Mg}$ ! $\mathrm{Fe}^{t}$ ratio. This indicates that more iron can be in the trivalent state.

\section{Glass inclusions}

General. During the crystallization small amounts of magma can become trapped in the crystals. It is of interest to know the composition of those glass inclusions because they can give information about the orig:na? 
magma and possibly the conditions where fractional crystallization or partial melting took place.

In the glass inclusions which usually consist of glass and a gas phase (Fig. 14) there are some variation in chemistry between inclusion and host magma. In olivine crystals the inclusions consist of a glass phase and a small gas bubble. In some of these inclusions traces of pyroxene and spinel are detected. In plagioclase crystals there is no evidence of similar crystal growth and there is no gas phase present in the inclusions.

Almost all the entrapped glass inclusions have composition intermediate between the groundmass glass phase and the whole rock chemistry, indicating that they are formed from similar magma.

Using Anderson's (1973) method to estimate the water content in possible original magma using the glass inclusion chemistry, some of the basaltic liquids were initially almost dry, but most of them had about $0.5-1.5 . \%$ water content.

Glass inclusions in olivines. The $\mathrm{MgO}$ content of the inclusions in the olivine microphenocrysts is lower $(0.1-0.4 \mathrm{wt-} \%)$ than in the surrounding glass. This is caused by the diffusion rate which is fast between the glass inclusion and olivine at relative high temperature. This leads mainly to the $\mathrm{CaO}$ and $\mathrm{FeO}$ enrichment in glass inclusions. There is no evidence of significant $\mathrm{MgO}$ increase near the crystal-inclusion interface. This is not in good agreement with the study of Anderson and Wright (1972).

In larger olivine macrophenocrysts similar $\mathrm{CaO}$ and $\mathrm{FeO}$ enrichment in glass inclusion can be obtained but in ever-increasing amounts. This increase is very marked for $\mathrm{TiO}_{2}$ as would be expected with the fractional crystallization.

However, in highly olivine normative (primitive) tholeiites there is no significant difference in chemistry between the glass phase

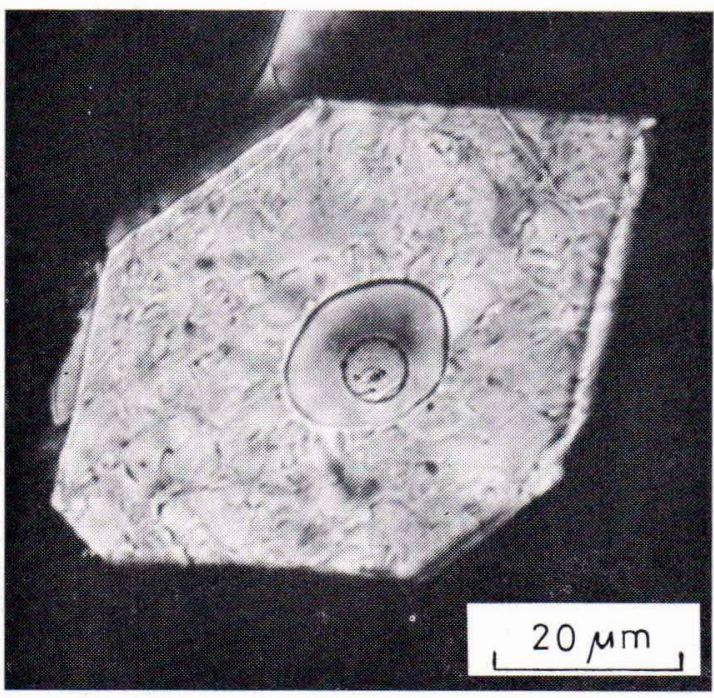

Fig. 14. Glass inclusion (glass and gas phases) in olivine (RE-17).

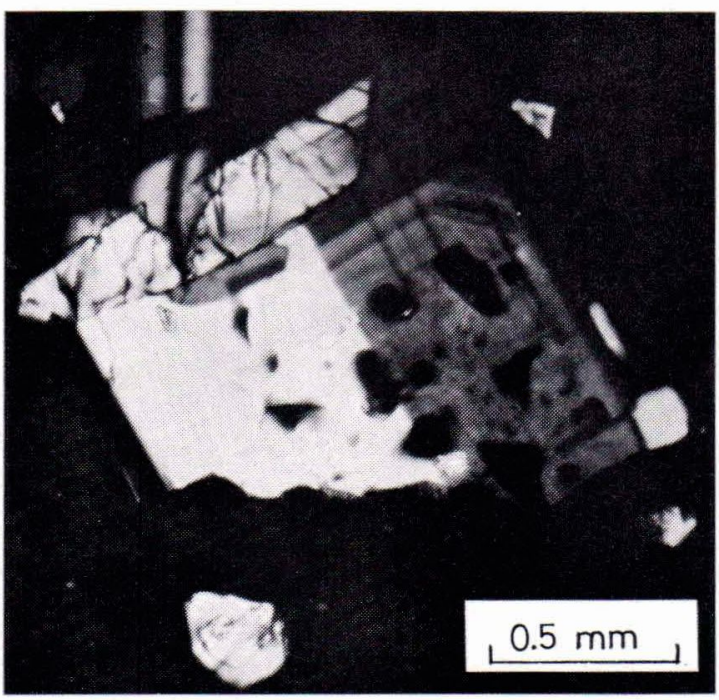

Fig. 15. Window-like growth in glass inclusions in plagioclase (RE-11).

and inclusions in phenocrysts. This might be due to high temperature and fast diffusion rate between the glass and crystal.

Glass inclusions in plagioclases. In the small plagioclase phenocrysts similar chemical effect as described for olivine can be observed. The $\mathrm{CaO}$ is lower $(0.1-0.5 \mathrm{wt}-0)$ in the glass inclusions than in the surrounding 


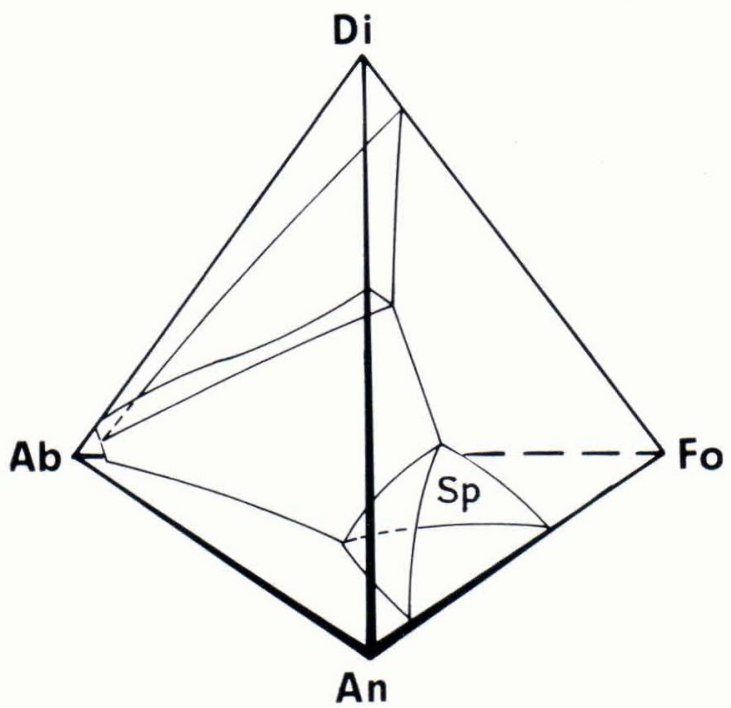

Fig. 16. Simple iron-free system for critically undersaturated basalts (Yoder and Tilley 1962, Fig. 10). Ab - albite, An - anorthite, Fo - forsterite, Di - diopside, Sp - spinel.

glass. In strong zoned phenocrysts the $\mathrm{CaO}$ enrichment is not effective and also $\mathrm{CaO}$ decrease can take place.

In large unzoned plagioclase phenocrysts the inclusion chemistry is very different from the surrounding glass chemistry. These latter ones may be xenoliths. (In the macrophenocrysts the window-like growth in the inclusions is the most widespread type (Fig. 15).)

\section{Discussion-conclusions}

All glasses and coexisting minerals are very homogenous within individual samples. Thin depletion zones, however, are found around phenocrysts. The glass surrounding olivines is depleted in $\mathrm{Mg}$ and $\mathrm{Fe}$ and is enriched in $\mathrm{Ca}$ and $\mathrm{Al}$ in a zone extending about $15 \mu \mathrm{m}$ from the olivine-glass interface. Plagioclase phenocrysts are in most cases surrounded by a zone depleted in $\mathrm{Ca}$ and $\mathrm{Al}$.
These observations suggest that phenocrysts crystallized largely in situ.

The system Ab-An-Fo-Di

In the crystallization study of basalts the system Ab-An-Fo-Di (Fig. 16) is used as a simple model for the understanding of crystallization processes. This tetrahedron includes the primary phases of basalts, namely olivine, plagioclase and clinopyroxene. The main limitation of this system is that it is calculated only in terms of $\mathrm{Na}_{2} \mathrm{O}, \mathrm{CaO}, \mathrm{MgO}$, $\mathrm{Al}_{2} \mathrm{O}_{3}$ and $\mathrm{SiO}_{2}$. Such important components as $\mathrm{FeO}, \mathrm{Fe}_{2} \mathrm{O}_{3}$ and $\mathrm{TiO}_{2}$ are absent. However, it is clear that such components as $\mathrm{Fe}_{2} \mathrm{O}_{3}$ and $\mathrm{TiO}_{2}$ have not very important effect to the main crystallization because they form principally accessory minerals, mainly ilmenite and magnetite. But $\mathrm{FeO}$ enters the pyroxenes and olivines lowering their liquidus temperature.

However, this system provides a convenient model for discussing the crystallization processes of basalts. The tetrahedron surface consists of four different three-component systems of which the two most important are briefly discussed.

The sub-system An-Di-Fo

This system (Fig. 17a) is of primary importance because it includes all major phases of tholeiites. The system was studied by Osborn and Tait (1952) who found that the system has two piercing points and a spinel field where spinel crystallizes as the primary phase.

The join Fo-Di is binary and according to Schairer and Yoder $(1961,1962)$ the eutectic point moves slightly toward the diopside corner with increasing water pressure (falling temperature). All other joins are discussed in the light of Ab-An-Di system. 

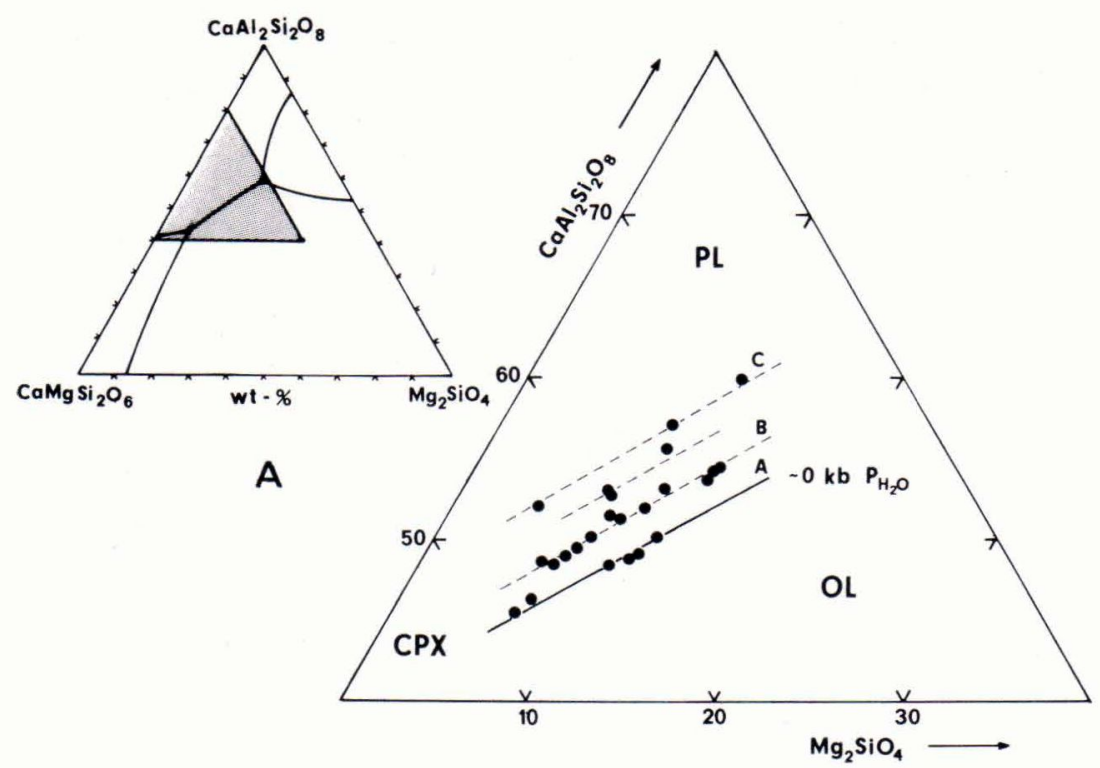

B

Fig. 17. Plot of the glass analyses (Table 2) from the Ab corner on the equilibrium diagram of the system anorthite-diopside-forsterite. The $0 \mathrm{~kb}$ water pressure line A in Fig. B drawn from Osborn and Tait (1953). Other lines see text.

The reaction of spinel with the liquid at relatively high temperature to form anorthite and forsterite plays an important role during the early stage of crystallization. According to Kushirn (1972) increasing pressure in the system Ol-Pl-Qz affects the field of spinel and $\mathrm{Ca}$-poor pyroxene expanding them relative to those of anorthite and forsterite. He also found the same effect in the five-component system $\mathrm{Na}, \mathrm{Ca}, \mathrm{Mg}, \mathrm{Al}$ and $\mathrm{Si}$. However, all these experiments were carried out under dry conditions and therefore the role of the volatile components on the spinel crystallization is uncertain. It appears most likely that the spinel field expands with increasing water pressure.

The present data was calculated in the terms of $\mathrm{Na}_{2} \mathrm{O}, \mathrm{CaO}, \mathrm{MgO}, \mathrm{Al}_{2} \mathrm{O}_{3}$ and $\mathrm{SiO}_{2}$ in the system Ab-An-Di-Fo. The An-Di-Fo diagram (Fig. 17b) shows the variation between individual samples. As seen from the figure the analyses apparently group around three distinct parallel curves (A, B and C). As all the samples appear to have plagioclase and olivine cotectic, one explanation to the shift from the curve $A$ to curve $C$ is variable water pressure. It appears, specially from Yoder's (1965) experiments, that rising water pressure expands olivine, spinel and clinopyroxene fields relative to the plagioclase field moving the cotectic curve toward the plagioclase corner.

The sub-system Ab-An-Di

This haplobasaltic system (Bowen 1915) is frequently used to show the crystallization processes of basaltic magmas. Although the system has one boundary curve it is not as simple as previously considered.

Osborn (1942) and Hytönen and Schairer (1961) found that the join Di-An is not binary because of solid solution in diopside. Schairer 


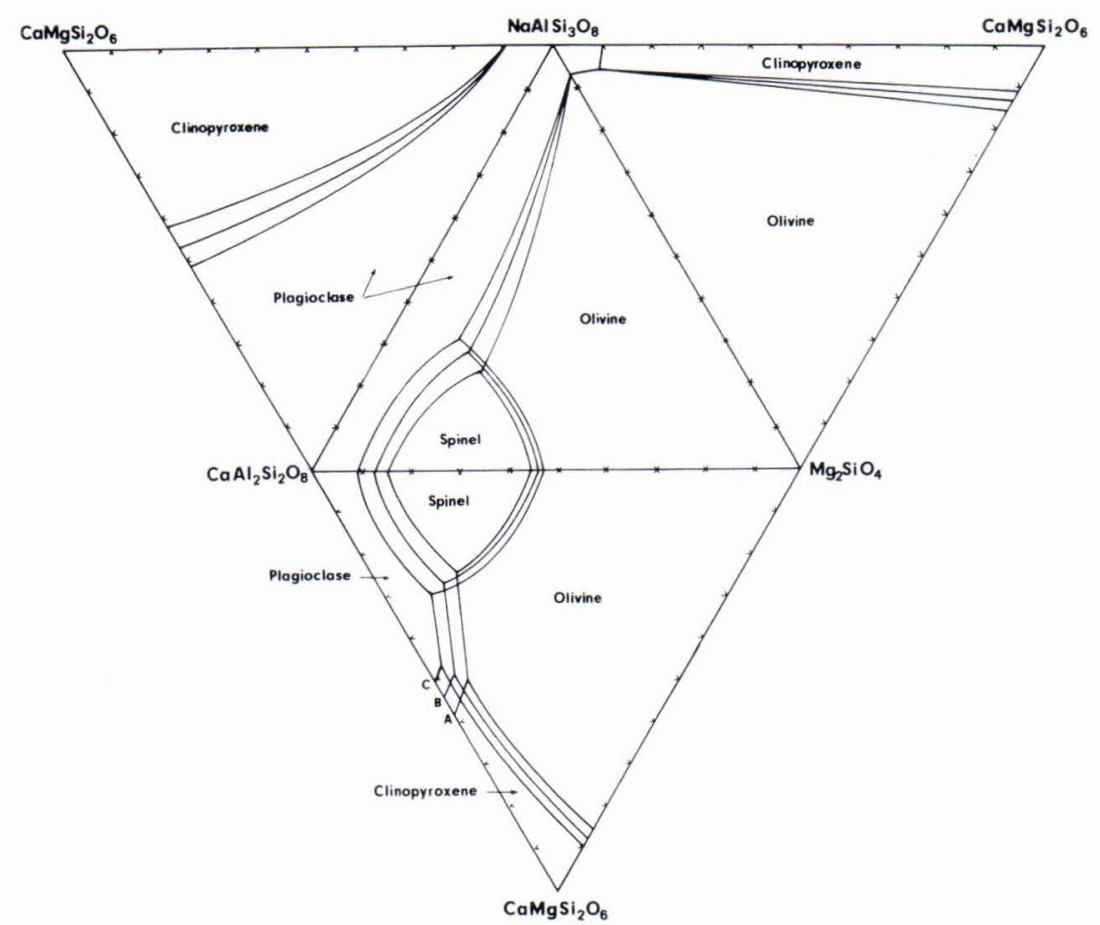

Fig. 18. The system diopside-albite-anorthite-forsterite laid out to show possible liquidus relations of the boundary systems. Lines A, B and C same as in Fig. 17.

and Yoder (1960) also found that the join Ab$\mathrm{Di}$ is not binary because of solid solution in plagioclase and that the piercing point between diopside and Ab-rich plagioclase is located at $\mathrm{Di}_{10} \mathrm{Ab}_{90}$ having about $7 \%$ more diopside than estimated previously by Bowen (1915). The boundary curve at 1 atm (Fig. 18) is drawn on the basis of more recent suggestions by Kushiro (1973).

The results on the basis of the basaltic Ab-An-Di-Fo tetrahedron suggest that these basalts on the present crystallization level would only fractionate olivine and plagioclase, but not clinopyroxene. However, clinopyroxene seems also to occur as phenocryst in some of these basalts. Some of these clinopyroxenes are autholiths formed at greater depths, because the clinopyroxene stability field expands at higher pressures.

However, the most successful result is that the different diagrams in this system show very clearly variation in water pressure conditions between individual samples. These results are in surprisingly good agreement with the estimations of original water contents on the basis of glass inclusion chemistry. Those basalts which have the highest assumed water pressure also have the highest water content. This does not necessarily mean that the total pressure should be different.

On the basis of this limited data it is of interest to note that these 'high water pressure' samples can be regionally related. Grimsvötn, Kerlingarfjöll and Thorisjökull massif (only the lowermost stratigraphical part) represent the highest assumed water pressure areas in Central Iceland (Localities refer to the sample numbers 22,24 and 96 , respectively, in Fig. 5). 
The system CMAS

The results derived from the system $\mathrm{Ab}-$ An-Di-Fo especially on the basis of different water pressures can also be caused by different iron and titanium contents in individual samples. For this reason the calculations were also made on the basis of more relevant CMAS-system (Fig. 19).

This system was introduced by O'Hara (1968) and several projections in this system are discussed in detail by O'Hara $(1970,1976)$. The calculations in this system are on the basis of all major elements:

$$
\begin{aligned}
\mathrm{C}= & \text { (mol.prop. } \mathrm{CaO}-31 / 3 \mathrm{P}_{2} \mathrm{O}_{5}+2 \mathrm{Na}_{2} \mathrm{O}+ \\
& \left.2 \mathrm{~K}_{2} \mathrm{O}\right) \times 56.08 \\
\mathrm{M}= & (\mathrm{mol} \text {.prop. } \mathrm{FeO}+\mathrm{MnO}+\mathrm{NiO}+\mathrm{MgO}- \\
& \left.\mathrm{TiO}_{2}\right) \times 40.31 \\
\mathrm{~A}= & \left(\mathrm{mol} \text {.prop. } \mathrm{Al}_{2} \mathrm{O}_{3}+\mathrm{Cr}_{2} \mathrm{O}_{3}+\mathrm{Fe}_{2} \mathrm{O}_{3}+\right. \\
& \left.\mathrm{Na}_{2} \mathrm{O}+\mathrm{K}_{2} \mathrm{O}+\mathrm{TiO}_{2}\right) \times 101.96 \\
\mathrm{~S}= & \left(\mathrm{mol} \text { prop. } \mathrm{SiO}_{2}-2 \mathrm{Na}_{2} \mathrm{O}-2 \mathrm{~K}_{2} \mathrm{O}\right) \times \\
& 60.09
\end{aligned}
$$

This system provides various possibilities using different sub-projections. The objective of the mode of the projection used here is to present the results in a form directly comparable with weight per cent plots and projections of the known phase equilibrium in the system $\mathrm{CaO}-\mathrm{Al}_{2} \mathrm{O}_{3}-\mathrm{MgO}-\mathrm{SiO}_{2}$.

It is meaningful to choose such sub-projection in this system which include all the major crystal phases of tholeiitic basalts. Sub-projection OL-OPX-CPX-PL (Fig. 20) includes all these phases. The cotectic surfaces in this tetrahedron are drawn on the basis of 30 experiments by $\mathrm{S}$. Steinthorsson (unpubl. data) and 8 experiments of the author using different types of Icelandic basalts. All experiments were carried out in dry conditions at 1 atm pressure.

Because the cotectic surfaces by chance are in difficult positions in the tetrahedron space, the plotting from each corner on different three component diagrams leads to large scatter. To minimize this effect the

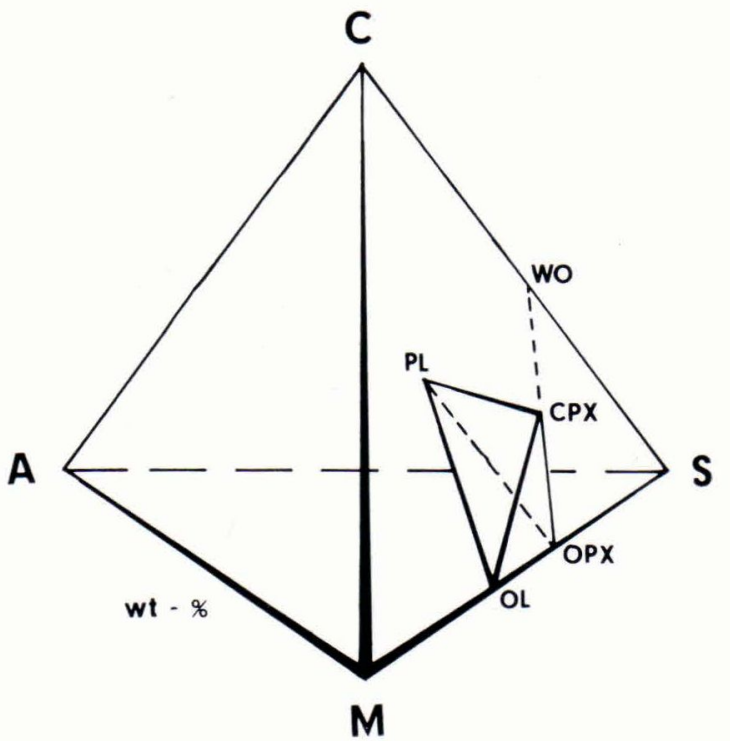

Fig. 19. The system CMAS (explanations see text). The tholeiitic basalt sub-projection plagioclase (PL) - olivine (OL) - orthopyroxene (OPX) clinopyroxene (CPX) is shown.

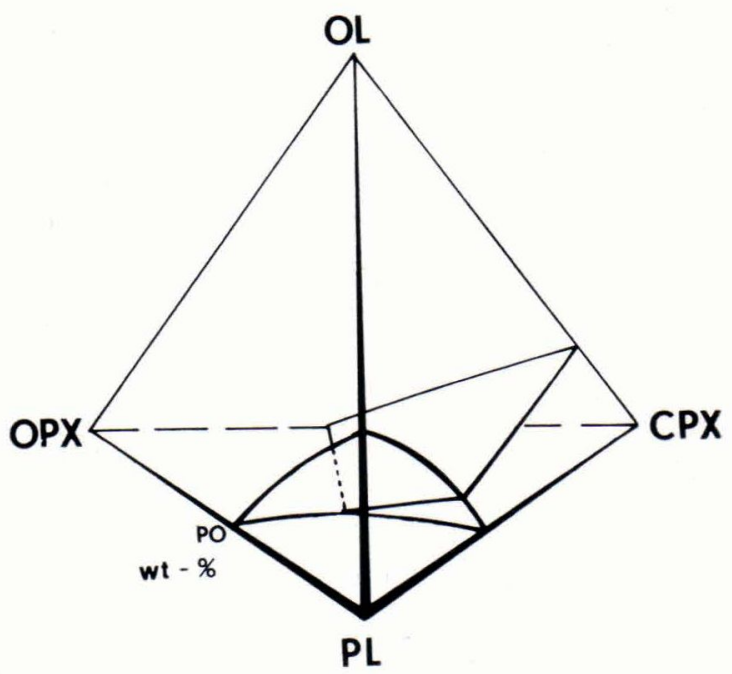

Fig. 20. The sub-projection OL-OPX-CPX-PL (see Fig. 19). The cotectic surfaces drawn using the present experimental data. $\mathrm{PO}$ is the projection point on the plane PL-OL-CPX.

plotting from point $\mathrm{PO}$ on the plane OL-PLCPX is chosen (Fig. 21). This point is chosen because all the samples which have PL, OL and CPX cotectic plot on the same point on 


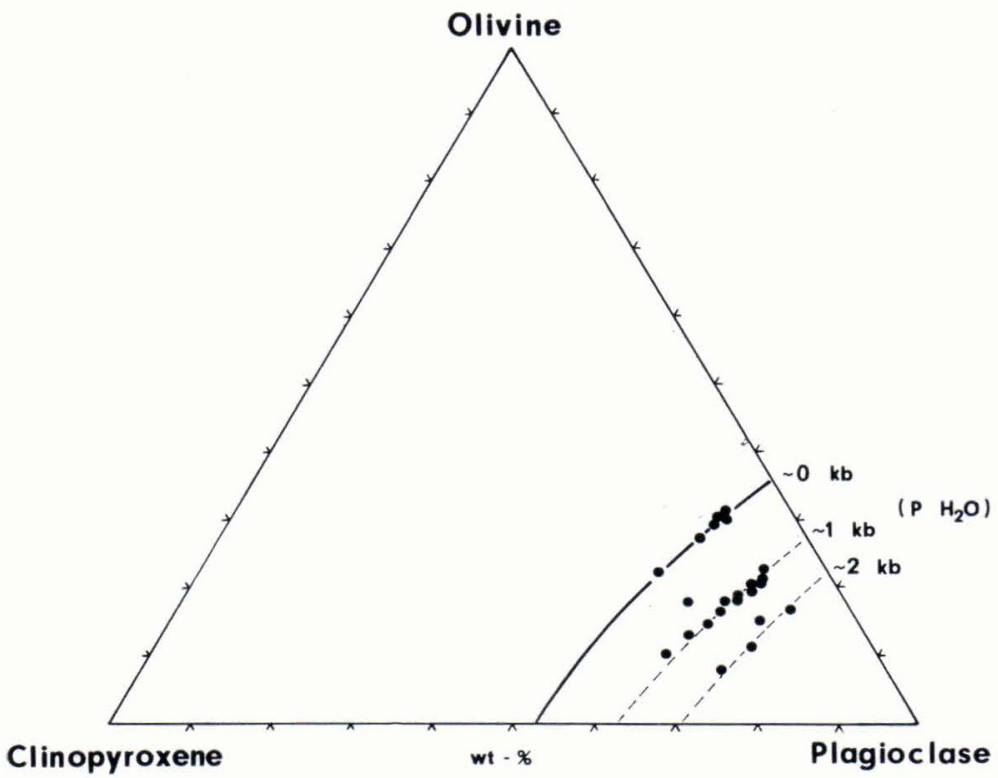

Fig. 21. Plot of the analyses from the point PO (Fig. 20) on the plane OL-CPX-PL in the sub-projection OL-CPX-OPXPL in the system CMAS. The $0 \mathrm{~kb}$ water pressure line drawn using present experimental data. Other lines see text. the plane OL-PL-CPX, and from this point all the analyses of this kind basalts can be plotted on this diagram.

All the samples are distinguishable in a similar way as in the system Ab-An-Di-Fo. According to O'Hara's (1968) water pressure experiments on the basis of A-CS-CM diagram the curves A, B and C in Figs. 17b and 21 can represent approximately $\mathrm{O}, 1$ and 2 $\mathrm{kb}$ water pressures, respectively.

The experiments performed in this study plot exactly on the $0 \mathrm{~kb}$ curve.

\section{Trace elements}

If the chemical variation among the samples from each single area can be expressed in terms of fractional crystallization it is of interest to plot each trace element against an index of the degree of fractional crystallization, the $\mathrm{FeO}^{\mathrm{t}} / \mathrm{MgO}$ ratio. Increasing values of the ratio $\mathrm{FeO}^{\mathrm{t}} / \mathrm{MgO}$ indicate more fractionated samples (Fig. 22).
The positive correlations between the fractionation index and $\mathrm{Ti}, \mathrm{Mn}, \mathrm{Cu}$ and $\mathrm{Zn}$ as well as the negative correlations between this ratio and $\mathrm{Ni}, \mathrm{Co}$ and $\mathrm{Cr}$ indicate fractional crystallization involving olivine, plagioclase and perhaps pyroxene. The breaks in $\mathrm{Ni}$, Co and $\mathrm{Cr}$ trends indicate beginning of plagioclase crystallization. The vanadium contents show significantly low values in some samples indicating mainly strong pyroxene crystallization.

If fractional crystallization of olivine, plagioclase, spinel and clinopyroxene is the cause it would be expected that $\mathrm{Cr}$ and $\mathrm{Ni}$ are extracted from residual liquid. Therefore it is meaningful to compare those elements with an element which shows strong positive correlation on increase with differentiation.

On the basis of Ti-Ni-Cr triangle (Fig. 23) the samples studied form very strong differentiation trend within each geographical group. This trend also has fairly good correlation with $\mathrm{MgO}$. 
The transition metals studied are normalized to $\mathrm{C} 1$ carbonaceous chondrite as it shows their geochemical behaviour (Fig. 24). Some features emerge from this figure:

- The transition metal distribution in basalts studied follows remarkably well the chondrite normalized field of the ocean floor basalts.

- $\mathrm{Cu}$ and $\mathrm{Zn}$ trends do not follow the trend of the other transition metals, because they behave as incompatible element during the early fractionation stages.

- $\mathrm{Zn}$ values are lower than on the basalt field.

- Co values are slightly higher than on the basalt field probably indicating special feature of Icelandic basalts.

$\mathrm{MgO}$ variation diagram

$\mathrm{MgO}$ variation diagrams (Fig. 25) show the relationships between the different oxides in the rocks studied. The whole rock analyses are plotted with different symbols from the glass analyses to show the effect of crystallization. Two intermediate rocks from the Kerlingarfjöll area are also plotted to emphasize the trends.

The $\mathrm{CaO}$ plot shows a break approximately at $9 \% \mathrm{MgO}$. Before that break olivine is the only phase to crystallize and leads to the $\mathrm{CaO}$ enrichment. $\mathrm{TiO}_{2}$ and $\mathrm{FeO}$ enriched approximately down to $4 \% \mathrm{MgO}$ and break down very rapid mainly because of the Fe-Ti oxides crystallization. The scatter in the Fe-Mg plotting results mainly from different amounts of olivine crystallization. $\mathrm{K}_{2} \mathrm{O}$ increases almost exponentially with decreasing of $\mathrm{MgO}$. This is what would be expected when the main crystallizing phases are olivine and plagioclase.

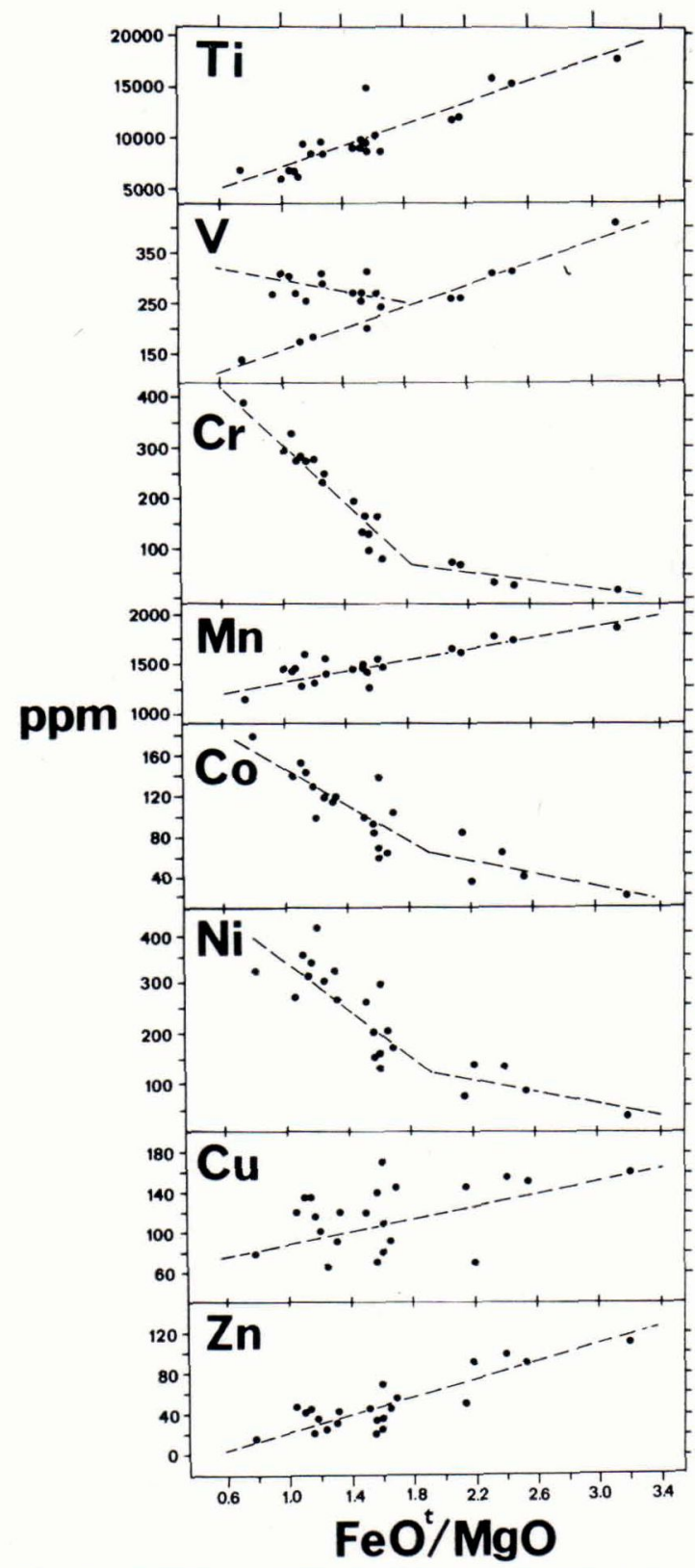

Fig. 22. Trace element variation against degree of fractional crystallization, $\mathrm{FeO}^{\mathrm{t}} / \mathrm{MgO}$.

\section{Model calculations}

The model calculations are done on the basis of the end members of separated spatial groups. These trends are shown in 

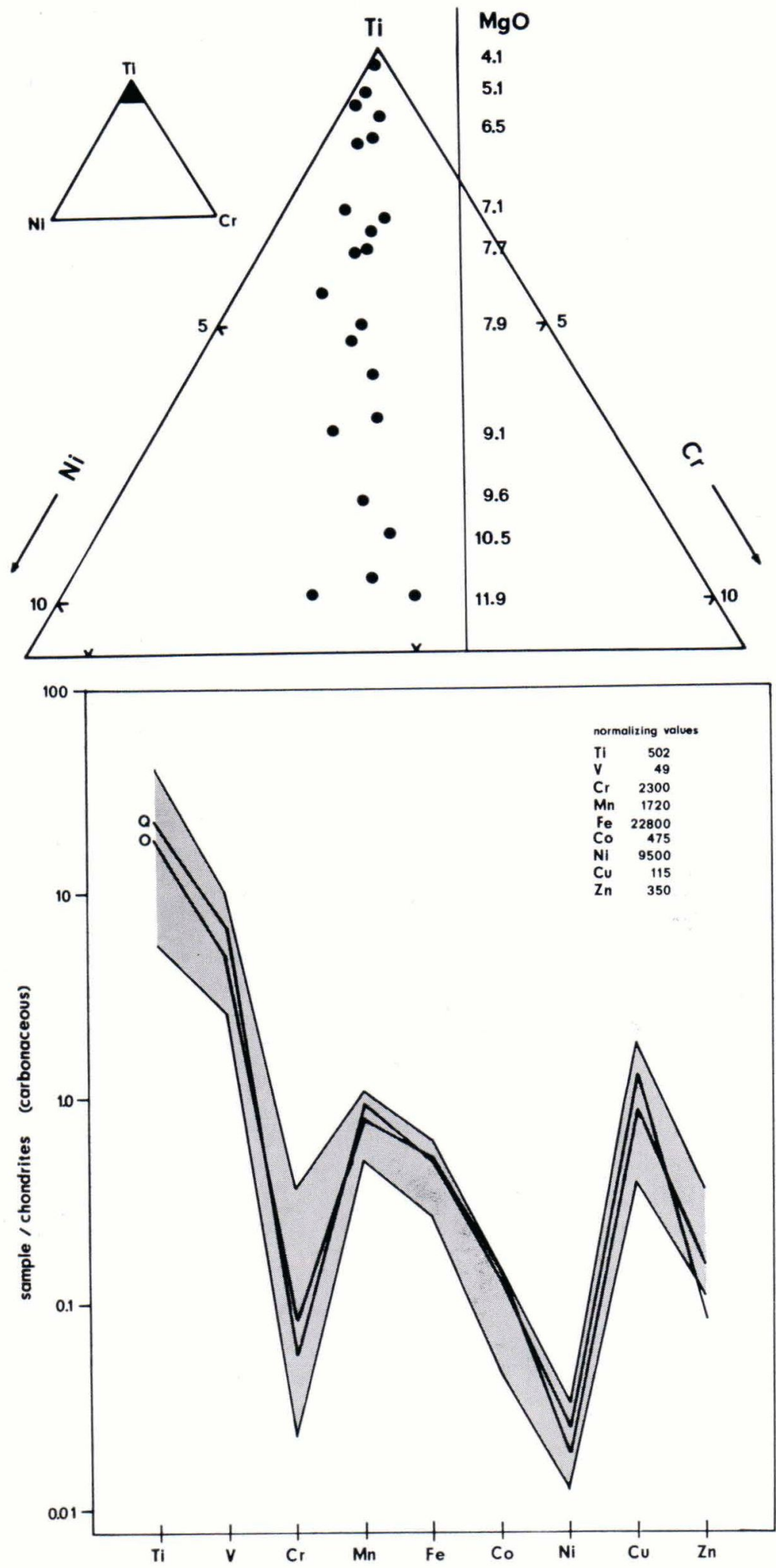

Fig. 23. Plot of the trace element analyses (Table 1) on the Ti-Ni-Cr triangle. The $\mathrm{MgO}$ variation in whole rocks is schematically shown.
Fig. 24. Chondrite-normalized transition metal distributions in studied basalts. Q - quartz normative tholeiites. $\mathrm{O}$ olivine normative tholeiites. The basalt field (shaded area) includes basalts from $45^{\circ} \mathrm{N}$ on the Mid-Atlantic Ridge (Muir et al. 1964), some Icelandic basalts (Sigvaldason 1974), basalts from Atlantic Ocean floor (Frey et al. 1974) and basalts from FAMOUS area (Langmuir et al. 1977). 
Fig. 7. The Theistareykir and Reykjanes peninsula trends are discussed in some details below.

The fractioned olivine and plagioclase compositions are assumed to be intermediate between that expected in the parential magma and in the residual liquid. The Theistareykir trend shows extreme variation against the index of the degree of fractional crystallization, $\mathrm{FeO}^{\mathrm{t}} / \mathrm{MgO} 1.67-1.21$, and the end members can be balanced with about 5.4 $\%$ olivine, $\mathrm{Fo}_{81}$ and $13 \%$ plagioclase, $\mathrm{An}_{78}$ fractionation. However, assuming that the fractionated minerals are more mantle-like olivine $\mathrm{Fo}_{90}$ (O'Hara et al. 1975) and plagioclase more $\mathrm{An}$ rich, $\mathrm{An}_{85}$, the corresponding balance fractionation values are $4 \%$ and 10 $\%$ respectively.

The Reykjanes peninsula trend shows large scatter in Fig. 7, but the end members (RE-19, RE-17) can be balanced only with $7.5 \%$ olivine, $\mathrm{Fo}_{81}$, fractionation.

\section{Summary}

1. The chemistry of twenty-two subglacial basalts from eastern and western volcanic zones of Iceland follow clearly the geopraphical deviation pattern of postglacial rocks.

2. The minerals found as phenocrysts are olivine, plagioclase, clinopyroxene and spinel.

3. In general all the microphenocrysts appear to be in equilibrium with the coexisting magma.

4. The glass inclusions in olivine and plagioclase crystals with similar chemistry to whole rock composition indicate that phenocrysts are formed in similar magma as they are now found.

5. The morphology and size of the crystals show that the main crystallization took

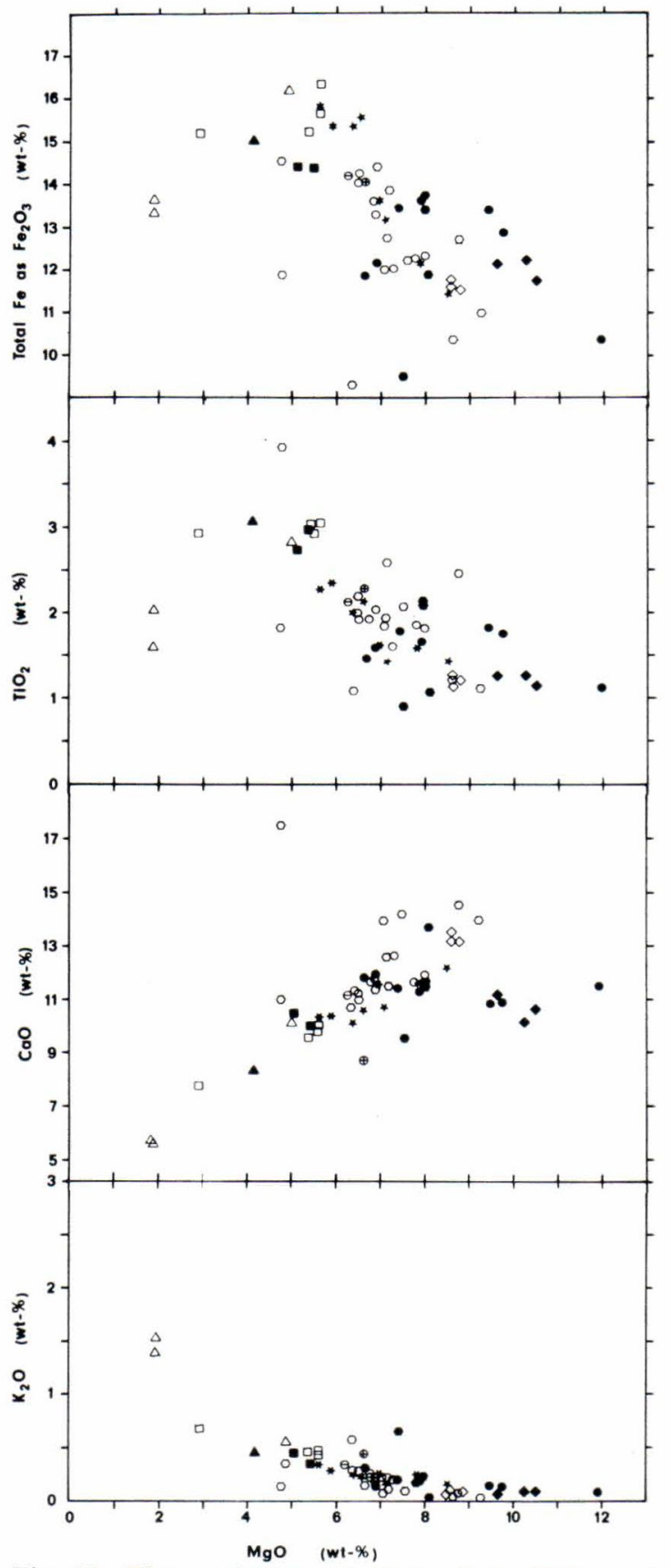

Fig. 25. The variation of $\mathrm{K}_{2} \mathrm{O}, \mathrm{CaO}, \mathrm{TiO}_{2}$ and $\mathrm{Fe}_{2} \mathrm{O}_{3}{ }^{\mathrm{t}}$ as a function of $\mathrm{MgO}$ (wt. ${ }^{\%}$ ). Filled symbols indicate whole rock analyses (Table 1) and open symbols glass analyses (Table 2). Symbols: Hestfjall (No. 30 in Fig. 5). - Reykjanes peninsula (No. 5 in Fig. 5). - Grimsvötn (No. 22 in Fig. 5). - Kerlingarfjöll (No. 24 in Fig. 5). $\star$ - Theistareykir area (No. 3 in Fig. 5). $\bigcirc-$ Thorisjökull area (No. 96 in Fig. 5). 
place at least in two events. The first crystallization began after the magma was injected into a magma chamber or chambers within Icelandic crust producing the large macrophenocrysts. The $\mathrm{P}-\mathrm{T}$ conditions were almost constant and the cooling rate of the magma is expected to have been very slow. The second crystallization took place just before the eruption and the smallest microphenocrysts formed at an early stage during ascent. The cooling rate of the magma is assumed to be order about 20 $40{ }^{\circ} \mathrm{C} / \mathrm{h}$ (Grove and Walker 1977).

6. The strong zoning in large olivine and plagioclase crystals is concluded to be cause of effective magma convection in the magmatic chambers.

7. The major and trace element data suggest that the magma variation in the same area is caused by strong fractional crystallization.

8. The transition metal patterns in the basalts studied follow very closely the chondrite normalized plot of other ocean floor basalts indicating that the basalts studied cannot be produced from a chondritic source regions.

9. The model calculations demonstrate that basalts from the same area can be genetically evolved from each other in terms of fractional crystallization. The chemi- cal difference between the extreme end members of each spatial group need only 5 olivine $\left(\mathrm{Fo}_{81}\right)$ and $13 \%$ plagioclase $\left(\mathrm{An}_{78}\right)$ fractionation to be balanced.

10. The studies in the different basaltic systems show variation between individual samples on the basis of different pressure conditions. The water pressure derived is concluded to vary regionally.

11. The water content estimations of different original magma types and the pressure trends observed agree well with the fact that the solubility of water in magmas increases with increasing pressure.

12. According to the experiments of Yoder and Tilley (1962) and O'Hara (1968) in different basaltic systems, it appears that $\mathrm{P}_{\text {tot }}>\mathrm{PH}_{2} \mathrm{O}$, and the total pressure of the main crystallization does not exceed $2 \mathrm{~kb}$ $(6-7 \mathrm{~km})$.

Acknowledgements - This study was carried out during the authors fellowship at the Nordic Volcanological Institute, Reykjavik, Iceland. The staff of the Institute assisted in many ways. Personally I wish to express my gratitude to Dr. Karl Grönvold for much valuable advice and guidance during the course of this work. Drs. Gudmundur E. Sigvaldason, Atso Vorma and Karl Grönvold critically reviewed the manuscript and made a number of useful suggestions. This is gratefully acknowledged.

\section{References}

Albee, A. and Ray, L. (1970) Correction factors for electron probe microanalysis of silicates, carbonates, phosphates and sulfates. Anal. Chem. 42: 1408-1414.

Anderson, A. T. (1973) The before-eruption water content of some high-alumina magmas. Bull. Volcan. 37: 530-553.

- and Wright, T. L. (1972) Phenocrysts and glass inclusions and their bearing on oxidation and mixing of basaltic magmas, Kilauea volcano, Hawaii. Am. Miner. 57: 188-216.
Bemmelen, R. N. van and Rutten, M. G. (1955) Tablemountains of Northern Iceland. Leiden. $217 \mathrm{p}$.

Bence, A. E. and Albee, A. L. (1968) Empirical correction factors for the electron microanalysis of silicates and oxides. J. Geol. 76: 382403.

Bowen, N. L. (1915) The crystallization of haplobasaltic, haplodioritic and related magmas. Am. J. Sci. 4th Ser. 40: 161-185.

Bryan, W. B. (1971) Mineralogical studies of sub- 
marine basalts. Carnegie Inst. Washington Yearb. 71: 369-403.

- (1972) Morphology of quench crystals in submarine basalts. J. Geophys. Res. 77: 58125819.

- and Kullerud, G. (1969) Meteorites. Carnegie Inst. Washington Yearb. 69: 245-249.

Carmichael, I. S. E. (1967) The mineralogy of Thingmuli, a Tertiary volcano in eastern Iceland. Am. Miner. 52: 1815-1841.

Deer, W. A., Howie, R. A. and Zussman, J. (1963) Rock Forming Minerals, Vol. 2, Chain silicates. Longmans, London.

Einarsson, Th. (1960) Geologie von Hellisheidi. Sonderveröffentlichungen des Geologischen Institutes der Universität Köln, 5 .

- (1968) Jardfrædi - Saga bergs og lands. Mál og Menning, Reykjavik. 335 p.

Einarsson, $\mathrm{Tr}$. (1965) On the geology of Stapafellsulur and the surrounding area. Soc. Sci. Islandica, Gr. 4, 1.

Frey, F. A., Bryan, W. B. and Thompson, G. (1974) Atlantic Ocean floor; geochemistry and petrology of basalts from Legs 2 and 3 of the Deep Sea Drilling Project. J. Geophys. Res. 79: 5507 -5527 .

Furnes, H. (1974) Volume relations between palagonite and authigenetic minerals in hyaloclastites, and its bearing on the rate of palagonitization. Bull. Volcan. 38: 173-186.

- (1975) Experimental palagonitization of basaltic glasses of varied composition. Contr. Mineral. Petrol. 50: 105-113.

Green, D. H. and Ringwood, A. E. (1967) The genesis of basaltic magmas. Contr. Mineral. Petrol. 15: 103-190.

Grönvold, K. (1972) Structural and petrochemical studies in the Kerlingarfjöll region, Central Iceland. Unpublished Dr. Phil. thesis, University of Oxford.

Grove, T. L. and Walker, D. (1977) Cooling histories of Apollo 15 quartz normative basalts. Proc. Lunar Sci. Conf. 8th: 1501-1520.

Hafner, S. S., Virgo, D. and Warburton, D. (1971) Oxidation state of iron in plagioclase from lunar basalts. Earth Planet. Sci. Lett. 12: 159166.

Hoppe, H. J. (1941) Untersuchungen an Palagonittuffen und über ihre Bildungsbedingungen. Chemie der Erde 13, No. 4.

Hytönen, K. and Schairer, J. F. (1961) The plane enstatite-anorthite-diopside and its relation to basalts. Carnegie Inst. Washington Yearb. 59: $75-78$.
Imsland, P. (1978) Microprobe analyses, standards and analytical statistics (in prep.). Nordic Volcanological Institute, Reykjavik, Iceland.

Irvine, T. N. (1967) Chromian spinel as a petrogenetic indicator. Part II, Petrologic applications. Can. J. Earth Sci. 4: 71-103.

Jakobsson, S. P. (1971) Palagonitiseringen af tefraen på Surtsey (English summary). Nat. Hist. Museum, Reykjavik, Iceland.

- (1972) Chemistry and distribution pattern of recent basaltic rocks in Iceland. Lithos 5: 365 -386 .

Jones, J. G. (1969) Pillow lavas as depth indicators. Am. J. Sci. 267: 181-195.

- (1970) Interglacial volcanoes on the Laugarvatn region, southwest Iceland. J. Geol. 78: 127140.

Kjartansson, G. (1943) Arnesinga saga, Reykjavik, Iceland.

Kushiro, I. (1972) Determination of liquidus relations in synthetic silicate systems with electron probe analysis; the system forsteritediopside-silica at 1 atmosphere. Am. Miner. 57: 1260-1271.

- (1973) The system diopsode-anorthite-albite: Determination of compositions of coexisting phases. Carnegie Inst. Washington Yearb. 72: $502-507$.

Langmuir, C. H., Bender, J. F., Bence, A. E., Hanson, G. N. and Taylor, S. R. (1977) Petrogenesis of basalts from FAMOUS area: MidAtlantic ridge. Earth Planet. Sci. Lett. 36: 133 $-156$.

Löfgren, G. (1974) An experimental study of plagioclase crystal morphology: isothermal crystallization. Am. J. Sci. 274: 243-273.

Mason, B. and Allen, R. O. (1973) Minor and trace elements in augite, hornblende, and pyrope megacrysts from Kakanui, New Zealand. N. Z. J. Geol. and Geophys. 16: 935-947.

Muir, I. D., Tilley, C. E. and Scoon, J. H. (1964) Basalts from the northern part of the rift zone of the Mid-Atlantic Ridge. J. Petrol. 5: 409434.

Nayudu, Y. R. (1964) Palagonite tuffs (hyaloclastites) and products of post-eruptive processes. Bull. Volcan. 27: 1-20.

Noe-Nygaard, A. (1940) Subglacial volcanic activity in ancient and recent times. Folia Geogr. Danica 1, No. 2.

O'Hara, M. J. (1968) The bearing of phase equilibria studies in synthetic and natural systems on the origin and evolution of basic 
and ultrabasic rocks. Earth Sci. Rev. 4: 69133 .

- (1970) Upper mantle composition inferred from laboratory experiments and observation of volcanic products. Phys. Earth Planet. Interiors 3: $236-245$.

- (1976) Data reduction and projection schemes for complex compositions. Prog. in experimental petrology. Natural Environ. Res. Council. Ser. D, No. 6: 109-112.

- and Mercy, E. L. P. (1975) Garnet-peridotite, primary ultrabasic magma and eclogite; interpretation of upper mantle processes in kimberlite. Phys. and Chemistry of the Earth 9: 571604.

Osborn. E. F. (1942) The system CaSiO3-diopsideanorthite. Am. J. Sci. 240: 751-788.

- and Tait, D. B. (1952) The system diopsideforsterite-anorthite. Am. J. Sci. Bowen volume: $413-433$.

Peacock, M. A. (1926a) The geology of Videy, SW. Iceland: A record of igneous action in glacial times. Trans. Roy. Soc. Edin., vol LIV, Part II (No. 9).

- (1926b) The volcano-glacial palagonite formation of Iceland. Geol. Mag. 63: 385-399.

Pjetruss, H. (1900) The glacial palagonite-formation of Iceland. Scott. Geogr. Mag. XVI: $265-293$.

Quaide, W. (1972) $\mathrm{Fe}$ and $\mathrm{Mg}$ in bytowniteanorthite plagioclase in lunar basaltic rocks (abstract). In Watkins (ed.) Lunar Science-III. Lunar Sci. Inst. Contr. 88: 624-626.

Rittman, A. (1961) Volcanoes and their activity. Interscience Publishers. J. Wiley and Sons, New York.

Schairer, J. F. and Yoder, H. S. Jr. (1960) The nature of residual liquids from crystalli- zation, with data on the system nephelinediopside-silica. Am. J. Sci. 258A: 273-283.

- (1961) Crystallization in the system nephelineforsterite-silica at 1 atmosphere pressure. Carnegie Inst. Washington Yearb. 60: 141-144.

- (1962) The system diopside-enstatite-silica. Carnegie Inst. Washington Yearb. 61: 75-82.

Sigvaldason, G. E. (1968) Structure and products of subaquatic volcanoes in Iceland. Contr. Mineral. Petrol. 18: 1-16.

- (1974) Basalts from the centre of the assumed Icelandic mantle plume. J. Petrol. 15: 497524.

Simkin, T. and Smith, J. V. (1970) Minor element distribution in olivine. J. Geol. 78: 304-325.

Taylor, L. A., Kullerud, G. and Bryan, W. B. (1971) Opaque mineralogy and textural features of Apollo 12 samples and a comparison with Apollo 11 rocks. Proc. Lunar Sci. Conf. 2nd: $855-871$.

Thoroddsen, T. (1906) Island, Grundriss der geographie und geologie. Peterm. Geogr. Mitth., Erg. CLII-CLIII.

Tyrrel, G. W. and Peacock, M. A. (1926) The petrology of Iceland. Trans. Roy. Soc. Edinb., vol 60, Part I, No. 3: 51-76.

Wood, B. J. (1975) Mixing properties of Tschermacitic clinopyroxenes and their application to geobarometry. In Extended Abstracts: International Conference on Geothermometry and Geobarometry, Pennsylvania State University, Pennsylvania.

Yoder, H. S. Jr. (1965) Diopside-anorthite-water at five and ten kilobars and its bearing on explosive volcanism. Carnegie Inst. Washington Yearb. 64: 82-89.

- and Tilley, C. E. (1962) Origin of basalt magmas. J. Petrol. 3: 346-532.

Manuscript received, April 10, 1978.

\section{APPENDIX 1}

\section{Description of the samples}

\section{Reykjanes peninsula}

RE-1

Specimen is taken from Stapafell mountain which is situated within a fracture zone (Einarsson 1965). Two different pillow layers can be distinguished in the mountain separated by a thin tuff layer. The hand specimen is very fine grained and dark brown in colour. The bedding also is very clear. The only mineral visible is yellow-greenish olivine. 
RE-5

Specimen is taken from the Sveifluhals mountain. This hyaloclastite complex is mostly produced by subaquatic fissure eruptions and the distinguish between each structural phase is very clear. The hand specimen is fine grained and brown in colour. Olivine and plagioclase crystals are visible.

RE-11

Stakihnukur hill is a part of the Hellisheidi complex and according to Einarsson (1960) it is formed mainly from dolerite intrusions, which are partly covered by some holocene lava. The tuff in NW-side is fine grained and dark brown in colour. Small plagioclase and olivine crystals are visible in hand specimen.

RE-16

RE-17

The hill Sandfell south from Litli Meitill, Hellisheidi, is formed of fine grained slightly palagnitized hyaloclastite tuff. Its relative height is only $80 \mathrm{~m}$. RE-16 is from the SEpart of the hill and RE-17 from Wpart. Both of the samples are taken approximately $20 \mathrm{~m}$ above the basement. The hand specimen is yellowish-brown in colour and large white plagioclase and yellowish olivine crystals can be seen.

RE-19

$\mathrm{RE}-20$

Vifilsfell is a part of the large Blafjöll mountains chain which is partly covered by holocene rocks. RF-19 is taken near the basement and RE-20 from about $100 \mathrm{~m}$ higher level. The hyaloclastites look very fresh and they are dark brown in colour. Green olivine is the most abundant mineral and only few plagioclase crystals can be seen.

Theistareykir area

TH-3

TH-4

TH-7

TH-10

The specimens from the Theistareykir area represent different ridges or different tablemountains. They differ from each other both in mineralogy and in colour. The colour varies between brown and black. The minerals visible in the hand specimen are plagioclase or olivine or both of them. The rocks were collected by Dr. K. Grönvold.

Thorisjökull massif

TJ-96

TJ-99

TJ-101

TJ-104

The hole massif $(9 \times 7 \mathrm{~km})$ is covered by ice. The relative height of the mountain on the top of the ice is 750 $\mathrm{m}$. Samples were collected from the bottom of the hill within about $50 \mathrm{~m}$ intervals. The specimens taken from the same altitude from different part of the hill differ from each other sofar by colour as minerals. The colour ranges from light brown to black and also the grain size varies between individual samples. All specimens contain either white plagioclase or green olivine or both of them. 
Kerlingarfjöll area

KE-24

Specimen was taken in the middle of the Kerlingarfjöll volcanic complex described by Grönvold (1972). The hand specimen is brown in colour and no minerals are visible. Sample was collected by Dr. K. Grönvold.

\section{Hestfjall volcano}

HE-30

HE-32

HE-35

The mountain is a large remnant of the interglacial volcano. It has been active also in glacial times and the northern part of the hill is formed of fine grained brown hyaloclastite. Specimens were collected within about $50 \mathrm{~m}$ intervals, thus $\mathrm{HE}-30$ is from the top of the mountain and $\mathrm{HE}-32$ and HE-35 are from $100 \mathrm{~m}$ and $250 \mathrm{~m}$ lower levels, respectively. In all specimens there are only few minerals visible or none at all.
Landmannalaugar area

LA-38

Specimen is taken from a small unnamed hill south of the mountain Stora Kyling. The hand specimen is coarse grained and dark brown in colour. Large plagioclase crystals are visible. Further the specimen contains some small xenoliths of gabbroic composition. These xenoliths can be explained as contamination from near situated Torfajökull acid massif.

\section{Grimsvötn area}

GR-22

GR-23

Grimsvötn is an active volcanic depression in the middle of the Vatnajökull ice massif. The volcano has been very active also in historical times erupting approximately every 10 th year. GR-22 and GR-23 are taken from Svartibunki and Naggur mountains, respectively. They are black in colour and contain no visible minerals. The rocks were collected by Dr. K. Grönvold. 